\title{
Notch signaling dynamically regulates adult $\beta$ cell proliferation and maturity
}

\author{
Alberto Bartolome, ${ }^{1}$ Changyu Zhu, ${ }^{1}$ Lori Sussel, ${ }^{2}$ and Utpal B. Pajvani' \\ 'Department of Medicine, Columbia University, New York, New York, USA. ²Department of Pediatrics, University of Colorado, Denver, Colorado, USA.
}

\begin{abstract}
Notch signaling regulates differentiation of the pancreatic endocrine lineage during embryogenesis, but the role of Notch in mature $\beta$ cells is unclear. We found that islets derived from lean mice show modest $\beta$ cell Notch activity, which increases in obesity and in response to high glucose. This response appeared maladaptive, as mice with $\beta$ cell-specific-deficient Notch transcriptional activity showed improved glucose tolerance when subjected to high-fat diet feeding. Conversely, mice with $\beta$ cell-specific Notch gain of function ( $\beta$-NICD) had a progressive loss of $\beta$ cell maturity, due to proteasomal degradation of MafA, leading to impaired glucose-stimulated insulin secretion and glucose intolerance with aging or obesity. Surprisingly, Notch-active $\beta$ cells had increased proliferative capacity, leading to increased but dysfunctional $\beta$ cell mass. These studies demonstrate a dynamic role for Notch in developed $\beta$ cells for simultaneously regulating $\beta$ cell function and proliferation.
\end{abstract}

\section{Introduction}

$\beta$ Cell mass and function adapt to the insulin requirements of the organism to maintain euglycemia across a wide range of pathophysiology, such as insulin resistance, pregnancy, or aging (1). Molecular mechanisms that enable $\beta$ cell adaptation to these stressors are not yet fully understood; insufficient functional adaptation, including $\beta$ cell death and dedifferentiation, becomes clinically apparent with the onset of type 2 diabetes (T2D) $(2,3)$. With the continued increase in obesity, novel therapeutically tractable pathways that regulate $\beta$ cell adaptation are sought to reverse $\beta$ cell dysfunction in T2D.

Notch mediates cell-fate decisions via juxtacrine interactions between neighboring cells expressing Notch receptors (Notch1-4) and ligands (Jagged1/2, Dll1/3/4). Mice lacking the common transcriptional effector of all Notch signaling, $R b p j$, or other Notch pathway components are embryonically lethal, but even prior to lethality, Notch mutant mice showed abnormal pancreata $(4,5)$. In fact, both Notch gain and loss of function impaired $\beta$ cell differentiation $(6,7)$, underscoring the necessity of Notch signaling for normal development, but the potential role of Notch in the adult endocrine pancreas remained mostly unexplored, which was surprising with the subsequent identification of NOTCH2 as a T2D susceptibility locus (8).

We have recently shown that liver Notch activity is increased in diet-induced obesity (DIO) mouse models and in T2D patients (9) and contributes to excess hepatic glucose production and fatty acid synthesis $(10,11)$. These data led us to hypothesize that Notch may be similarly reactivated in the stressed $\beta$ cell, consistent with other "developmental" pathways in the dedifferentiated $\beta$ cell (3). Here, we find that Notch signaling is present at low levels in fully

Conflict of interest: The authors have declared that no conflict of interest exists. License: Copyright 2019, American Society for Clinical Investigation.

Submitted: October 17, 2017; Accepted: October 25, 2018.

Reference information: / Clin Invest. 2019;129(1):268-280.

https://doi.org/10.1172/JCI98098. developed $\beta$ cells, but increased in islets cultured in hyperglycemic conditions or isolated from obese mice. Persistent $\beta$ cell Notch signaling appears detrimental to function, as forced Notch activation impaired glucose-stimulated insulin secretion (GSIS) in isolated mouse or human islets and glucose intolerance in $\beta$ cell-specific Notch gain-of-function mouse models. Conversely, we observed improved glucose tolerance with genetic inhibition of $\beta$ cell Notch action. Mechanistically, we found that Notch interfered with MafA-Kat2b association, which induced MafA proteasomal degradation, loss of $\beta$ cell maturity, and surprisingly, a simultaneous $\beta$ cell proliferative response. These data suggest that Notch signaling acts as a switch controlling 2 diametrically opposed events maturity and proliferation - in adult $\beta$ cells.

\section{Results}

Notch signaling is dynamically regulated in developed pancreatic $\beta$ cells. As a first step to evaluating a potential postdevelopment role of $\beta$ cell Notch signaling, we determined the absolute expression of Notch signaling components in islets isolated from WT adult mice (Supplemental Figure 1A; supplemental material available online with this article; https://doi.org/10.1172/JCI98098DS1). This analysis revealed relatively high expression levels of Notch receptors/ligands in islets, consistent with prevalent Rbpj staining in $\beta$ cells (Figure 1A), suggesting potential for ongoing $\beta$ cell Notch signaling. To assess Notch activation in the adult pancreas, we used transgenic Notch reporter (TNR) mice that express GFP under the control of a Rbpj consensus sequence (12). Chow-fed TNR mice showed readily detectable Notch activity in a subset of $\beta$ cells (Figure 1B), but trivial staining in other islet endocrine cells (Supplemental Figure 1B). We also observed an enrichment of $\beta$ cell transcripts (i.e., Ins2) in FACS-sorted, Notch-active $\mathrm{GFP}^{+}$ islet cells (Supplemental Figure 1C). We next used another Notch reporter mouse model, with a more persistent fluorescent reporter (Histone2b-Venus) (13), to confirm these data - integrating both data sets, we found the islet Notch activity was essentially confined to $\beta$ cells (Supplemental Figure 1D), but at generally lower 
A

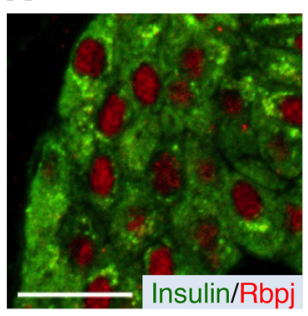

D
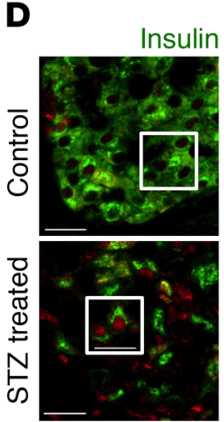

G

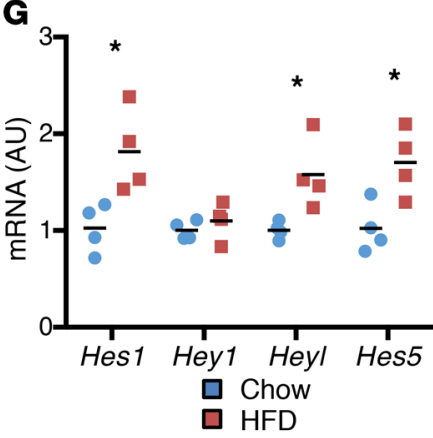

B

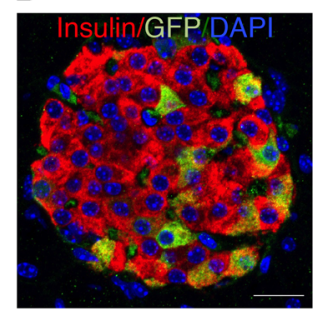

E

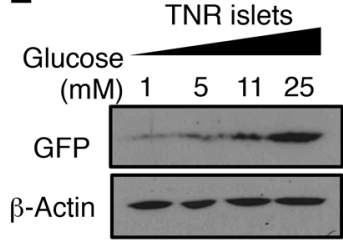

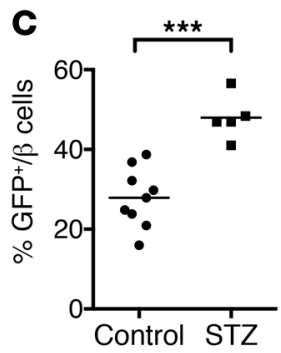

$\mathbf{F}$

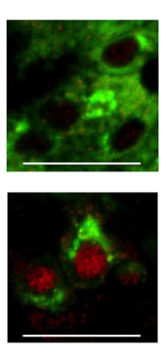

H

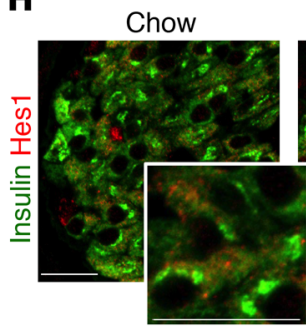

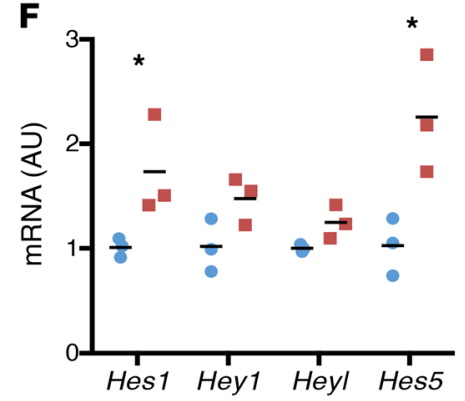

$\square$ Low glucose $\square$ High glucose

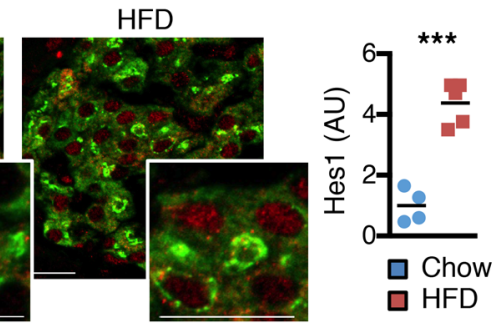

Figure 1. Increased Notch activity with $\boldsymbol{\beta}$ cell stress. (A) Representative image of pancreatic sections from WT mice stained with antibodies directed against insulin and Rbpj. (B) Representative image of pancreatic sections from TNR mice. (C) Quantitation of percentage of GFP ${ }^{+}$cells in remaining $\beta$ cells from TNR mice after low-dose STZ ( $n=5-9$ mice/group). (D) Representative images of pancreatic sections stained with antibodies directed against Hes1 and insulin in vehicle (control) and STZ-treated TNR mice ( $n=5-9$ mice/group). (E) Western blots from islets isolated from TNR mice, incubated for 15 hours in medium containing indicated glucose concentrations. Representative blots from 2 experiments. (F) Gene expression in islets isolated from WT mice, cultured overnight in medium containing low ( $1 \mathrm{mM}$ ) or high $(25 \mathrm{mM})$ glucose $(n=3$ biologic replicates). (G) Gene expression in islets isolated from $24-w e e k$ HFD-fed WT mice, as compared with normal chow diet-fed littermate controls ( $n=5$ mice/group). (H) Representative images of pancreatic sections stained with antibodies directed against Hes1 and insulin, with quantitation of nuclear Hes1 fluorescence intensity in $\beta$ cells ( $n=4-5$ mice/group). Scale bars: $20 \mu$ m. All data are shown with group means. ${ }^{*} P<0.05 ;{ }^{* *} P<0.001,2$-tailed $t$ test.

levels than Notch activity detected in endothelial or duct cells (Supplemental Figure 1E), consistent with earlier observations (14).

We next evaluated whether $\beta$ cell Notch activity was regulated by metabolic stimuli. We used low-dose streptozotocin (STZ) treatment to render TNR mice hyperglycemic, which increased $\beta$ cell Notch activity (Figure 1C and Supplemental Figure 1F). This was confirmed by increased expression of the canonical Notch target Hes1 in the surviving $\beta$ cell population (Figure 1D). We attributed increased Notch activity to hyperglycemia, as opposed to an STZinduced injury response, as high-glucose exposure also resulted in increased GFP protein levels and Hes1 expression in isolated TNR islets (Figure 1, E and F), consistent with increased Notch signaling in islets from hyperglycemic NOD mice (15). Similarly, we found higher expression of Hes1 and other Notch transcriptional targets in islets isolated from DIO mice, as compared with chowfed mice (Figure 1G), which corresponded with increased $\beta$ cell
Hes1 staining (Figure 1H). Thus, we conclude that Notch signaling is active in adult $\beta$ cells and enhanced in conditions associated with increased insulin demand (obesity, partial ablation of $\beta$ cell mass, and hyperglycemia).

$\beta$ cell Notch activation impairs GSIS, leading to glucose intolerance. To test whether Notch may contribute to $\beta$ cell dysfunction, we generated $\beta$ cell-specific Notch gain-of-function ( $\beta$-NICD) mice, which express the constitutively active Notch intracelluar domain (NICD), by crossing Rosa26-NICD (7) with RIP-Cre (16) transgenic lines. Despite unchanged body weight (Supplemental Figure 2A), chowfed $\beta$-NICD mice were mildly glucose intolerant as compared with $\mathrm{Cre}^{-}$controls (Figure 2A). These differences were exacerbated by aging (Figure 2B) or high-fat diet (HFD) feeding (Figure 2C), but completely reversed with simultaneous Rbpj deletion (Figure 2D), suggesting glucose intolerance was mediated by canonical Notch signaling. $\beta$-NICD mice showed unchanged fasting insulin levels 
(Supplemental Figure 2B), but blunted in vivo (Figure 2E) and ex vivo (Figure 2F) GSIS and a small decrease in total insulin content (Figure $2 \mathrm{G}$ ). Consistent with this observation, islets from $\beta$-NICD mice showed decreased expression of genes corresponding to the GSIS machinery, including Pcsk1, Pcsk2, and Slc30a8 (Figure 2H), lower Slc2a2 staining (Figure 2I), and fewer $\beta$ cell mature dense core insulin granules (Figure 2J).

To exclude the impact of Notch activation during the early postnatal period, we generated tamoxifen-dependent (TAMdependent), $\beta$ cell-specific (17) Notch gain-of-function (MIP$\beta$-NICD) mice. MIP- $\beta$-NICD mice were indistinguishable from controls before TAM treatment, but consistent with data from $\beta$-NICD mice, showed increased glucose excursion after TAM treatment that was exacerbated by HFD feeding (Supplemental Figure 2C). In sum, these data suggest that forced Notch activation in fully developed $\beta$ cells is sufficient to impair functional response to obesity-induced insulin resistance.

Notch activity leads to a specific loss of $\beta$ cell MafA in mouse and human islets. To determine how Notch affects $\beta$ cell function, we took a candidate approach, focusing initially on key transcriptional regulators of GSIS, such as Pdx1, MafA, and Nkx6.1. Of these, we found a profound reduction in MafA staining in pancreatic sections, islets, and primary $\beta$ cells from $\beta$-NICD- and TAM-treated MIP- $\beta$-NICD mice (Figure 3, A and B, and Supplemental Figure 3, A and B), but importantly, not in $\beta$-NICD:Rbpj mice (Supplemental Figure 3C). Notch-mediated loss of $\beta$ cell MafA appeared relatively specific, without effects on Pdx1 or Nkx6.1 (Figure 3C, Supplemental Figure 3D and not shown), and also did not lead to a compensatory response in MafB, which localizes in glucagon ${ }^{+}$cells equally in control and $\beta$-NICD mice (Supplemental Figure 3, E and F). Consistent with the hypothesis that decreased MafA is the mechanism of impaired GSIS with $\beta$ cell Notch activation, shRNA-mediated Mafa silencing failed to exacerbate Notch inhibition of GSIS (Supplemental Figure $3 G)$. Finally, NICD transduction in human islets also resulted in loss of MAFA (Figure 3D) and impaired GSIS (Figure 3E), as well as lower PDX1, but without changes in MAFB and NKX6.1 (Supplemental Figure 4, A-C).

Notch causes MafA degradation by preventing its association with $K a t 2 b$. Intriguingly, Notch activation reduced MafA protein without affecting Mafa expression (Supplemental Figure 5, A and B), suggesting a posttranscriptional effect. To test this hypothesis, we generated a stable MIN6 line to allow doxycycline-dependent expression of a Notch1 gain-of-function mutant $(\mathrm{N} 1 \Delta \mathrm{E})$ that does not require ligand binding, but still requires $\gamma$-secretase processing to render NICD (18). As hypothesized, N1 $\Delta \mathrm{E}$ led to loss of MafA (Figure 3F), but not in the presence of a $\gamma$-secretase inhibitor (GSI) (Supplemental Figure 5, C and D) or in cells that express dominant-negative mastermind-like 1 (DNMAML), which inhibits function of the MAML transcriptional coactivator essential for Rbpj-dependent Notch signaling (Supplemental Figure 5E). Using this model, we confirmed that acute Notch activation rapidly decreases MafA protein (Figure 3G) without change in Mafa mRNA (Figure 3H).

We next observed that MG132-mediated proteasome inhibition preserved MafA protein levels (Figure 4A), which led us to hypothesize that Notch increases GSK3 $\beta$ activity, known to trigger MafA proteasomal degradation $(19,20)$. N1 $\Delta \mathrm{E}$, however, did not affect GSK3 $\beta$ activation (Supplemental Figure 5F), although GSK3 $\beta$ inhibition prevented Notch-mediated MafA ubiquitination (Supplemental Figure 5G) and degradation (Supplemental Figure 5H). Similarly, whereas exogenous MafA was also degraded with Notch activation, the GSK3ß-resistant MafA-S65A mutant (21) was protected from Notch-induced degradation (Supplemental Figure 5I). These results suggest that Notch-induced proteasomal degradation of MafA is downstream of GSK3 $\beta$-mediated phosphorylation.

The histone acetyltransferase Kat $2 \mathrm{~b}$ has been reported as protecting MafA from proteasomal degradation in cancer cells (20); as Kat2b can also interact with NICD (22), we hypothesized that Notch may affect MafA-Kat2b interaction, leading to MafA degradation. Indeed, Notch activation prevented normal Kat2b-MafA association (Figure 4B), and Kat2b overexpression in MIN6 cells protected MafA from Notch-mediated degradation (Figure 4C). Conversely, Kat $2 b$ silencing in MIN6 cells induced loss of MafA (Figure 4, D and E) without changing Mafa expression (Supplemental Figure 5J) and recapitulated the impaired GSIS associated with Notch activation (Figure $4 \mathrm{~F}$ ). In sum, these data show that Notch disrupts MafA-Kat2b interaction, which induces MafA proteasomal degradation and impaired $\beta$ cell function (Figure $4 G$ ).

Notch activation and reduced maturity in proliferating $\beta$ cells. Profiling experiments have reported reduced gene expression of maturity markers in proliferating $\beta$ cells (23). In line with this idea, we observed lower MafA protein in BrdU ${ }^{+}$cells from WT pregnant females (Figure 5A and Supplemental Figure 6A), but normal Pdx1, Neurod1, or Ucn3 (Figure 5B). We found a similar effect in MIN6 cells, which lose MafA when undergoing chromatin condensation prior to cell division, but retain Pdx1 and Nkx6.1 (Supplemental Figure $6 \mathrm{~B}$ ). Consistent with a Notch-MafA axis to regulate $\beta$ cell maturity and proliferation, we observed increased Notch-active $\beta$ cells in pregnant females as compared with virgin females (Figure $5 \mathrm{C}$ ) as well as in the highly proliferative $\beta$ cells remaining after STZ treatment (Figure 1, C and D, and Supplemental Figure 6C). In fact, the population of $\mathrm{KI} 67^{+}$proliferating $\beta$ cells in pregnant TNR mice was enriched in $\mathrm{GFP}^{+}$cells (Figure 5D); consistently, MafA levels were modestly reduced in $\mathrm{GFP}^{+}$cells (Figure 5E).

These results suggest that a transient increase in Notch activity accompanies loss of $\beta$ cell maturity in the course of normal $\beta$ cell self-replication. We next asked whether persistent Notch activation causes loss of $\beta$ cell maturity - indeed, islets from $\beta$-NICD mice showed a transcriptional signature of immaturity (decreased Mafa, Glp1r, and Ucn3 and increased Ldha; Supplemental Figure $6, \mathrm{D}$ and E). Similar data were observed in TAM-treated MIP- $\beta$ NICD mice (Supplemental Figure 6F). Reduced Mafa and maturity in Notch-active $\beta$ cells could arise from a feed-forward loop of Maf transcription factors (24), as previously shown for Mafa (25), or because chronic metabolic abnormalities in Notch gain-offunction mice repressed Mafa. To distinguish these possibilities, we treated islets derived from MIP- $\beta$-NICD mice with chronic 4-hydroxytamoxifen (4-OHT) treatment, which also revealed reduced Mafa (Supplemental Figure 6, G and H). In sum, these data indicate that $\beta$ cell Notch activation destabilizes MafA, which in turn represses the $\beta$ cell maturation program, but does not result in complete loss of cell identity, as lineage-tracing experiments did 
A

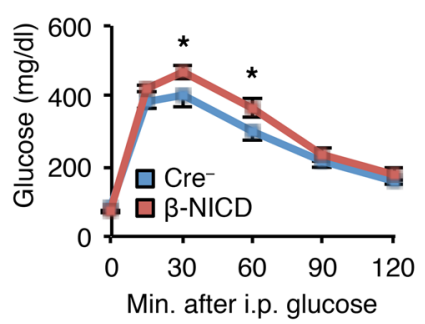

B

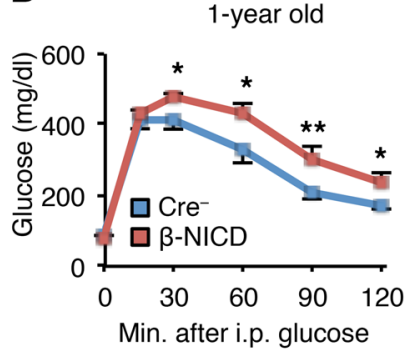

C

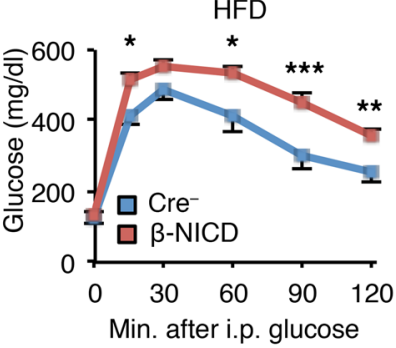

D

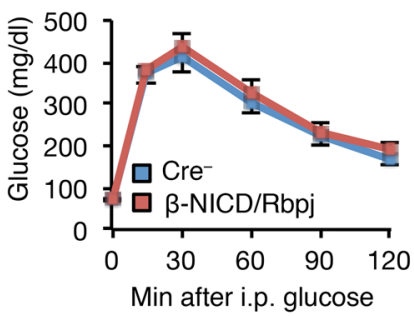

$\mathbf{E}$

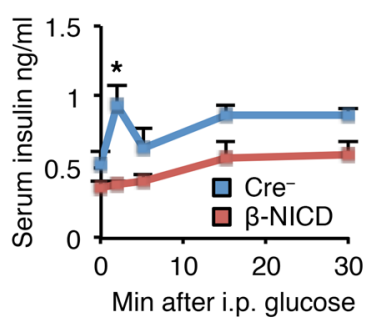

$\mathbf{F}$

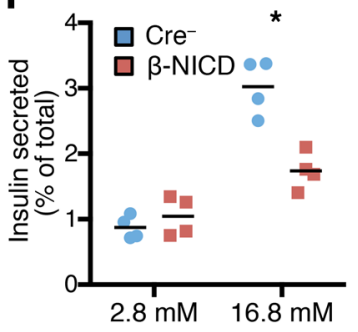

G

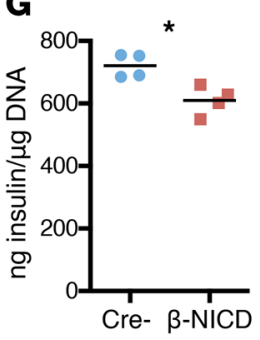

H

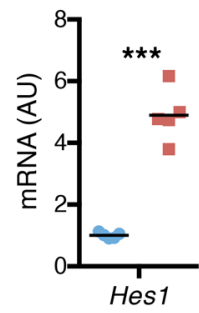

I
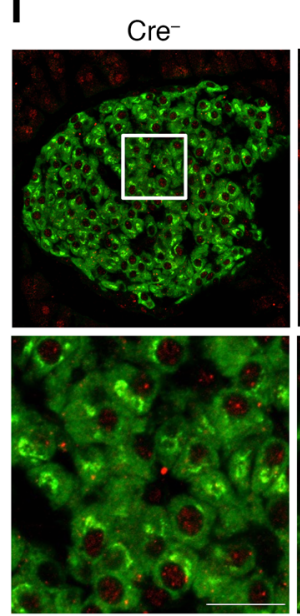

Hes1 Insulin
$\beta-N I C D$
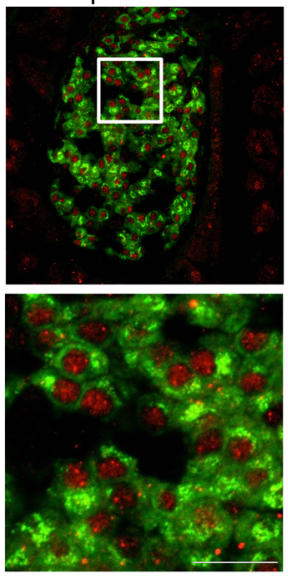
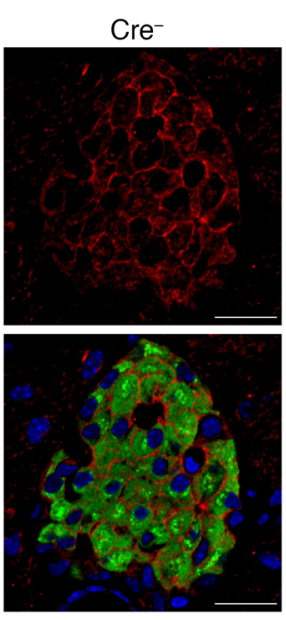

Slc2a2 Insulin DAPI
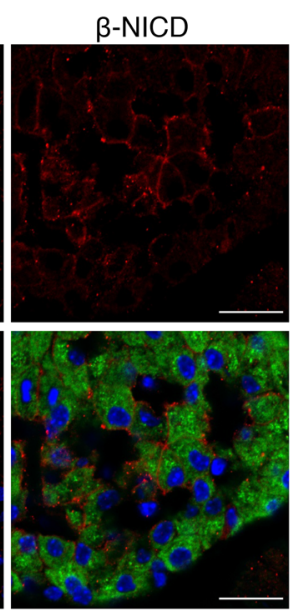

(1)

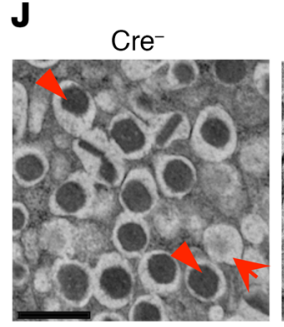

$\beta-N I C D$
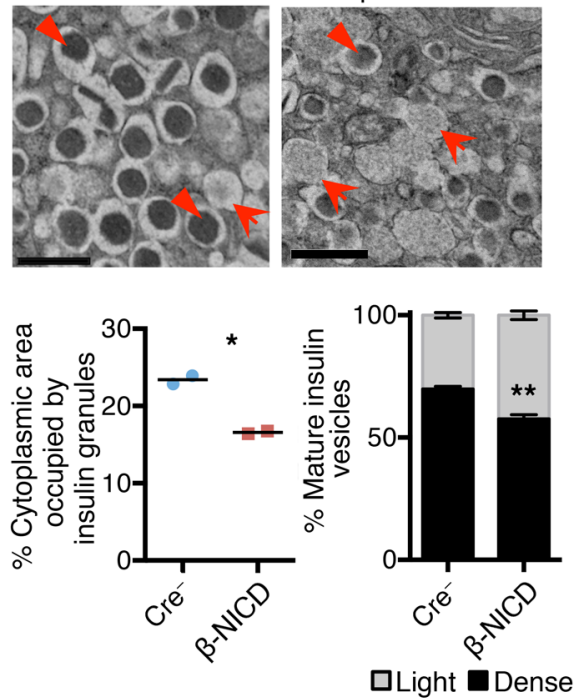

Figure 2. $\beta$ Cell Notch activation prevents normal GSIS, leading to glucose intolerance. (A) GTT in chow-fed, adult $\beta$-NICD, and Cre- control mice ( $n=7-8$ mice/group). (B) GTT in chow-fed, 1 year-old $\beta$-NICD mice and Cre- control mice ( $n=7-8$ mice/group). (C) GTT in $\beta$-NICD and Cre- control mice fed HFD for 8 weeks ( $n=8-10$ mice/group). (D) GTT in chow-fed, adult $\beta$-NICD/Rbpj and Cre- control mice ( $n=7$ mice/group). (E) Plasma insulin postintraperitoneal glucose injection in chow-fed $\beta$-NICD and Cre ${ }^{-}$control mice ( $n=5$ mice/group). (F) GSIS in islets isolated from $\beta$-NICD and Cre ${ }^{-}$control mice, adjusted for islet insulin content ( $n=4$ mice/group). (C) Insulin content in islets isolated from $\beta$-NICD and Cre- control mice, adjusted for islet DNA content ( $n=4$ mice/ group). (H) Gene expression in islets isolated from chow-fed $\beta$-NICD and Cre- control mice ( $n=5$ mice/group). (I) Representative images of pancreatic sections from $\beta$-NICD and Cre- control mice stained with antibodies directed against Hes1 (left) or SIc2a2 (right) ( $n=5$ mice/group). Scale bars: $20 \mu \mathrm{m}$. (J) Representative electron micrographs from 1-year-old $\beta$-NICD mice and Cre- control mice (top). Arrows represent immature vesicles, and arrowheads show mature vesicles. Scale bars: $500 \mathrm{~nm}$. Quantitation of mature (over total) dense-core insulin vesicles (bottom left) and cytoplasmic area occupied by insulin vesicles (bottom right) ( $n=2$ mice/group). All data are shown with group means $\pm \mathrm{SEM} .{ }^{*} P<0.05 ;{ }^{* *} P<0.01$; ${ }^{* *} P<0.001,2$-tailed $t$ test.

not reveal $\beta$ cell transdifferentiation or dedifferentiation in $\beta$-NICD mice (Supplemental Figure 6I).

Notch induces $\beta$ cell proliferation, leading to increased $\beta$ cell mass. The positive correlation between Notch activity and $\beta$ cell proliferation in TNR mice led us to test causation. We observed an increase in $\beta$ cell mass in $\beta$-NICD mice, due to a shift toward larger islet size (Figure 6A and Supplemental Figure 7A). We attributed increased $\beta$ cell mass in $\beta$-NICD mice to increased $\beta$ cell proliferation (Figure 6,
B and C), detectable as early as P14 (Figure 6D), as $\beta$ cell death was unchanged (Supplemental Figure $7 \mathrm{~B}) . \beta$ Cell mass remained higher in $\beta$-NICD mice even at 1 year of age (Figure $6 \mathrm{E}$ ), when $\beta$ cell proliferation rates were low and not different from those of control mice (Supplemental Figure 7C), suggesting that Notch-induced $\beta$ cell mass acquisition may be maintained through adulthood.

To determine the necessity of intact Notch signaling for proliferation, we generated $\beta$ cell-specific Notch loss-of-function 
A

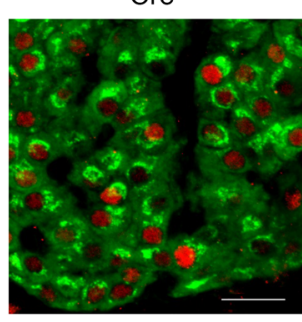

MafA Insulin

B

MafA

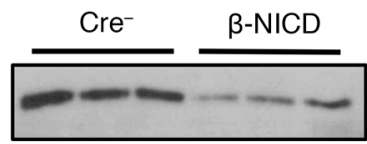

$\beta$-Actin

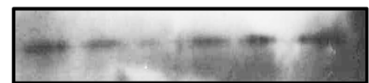

C

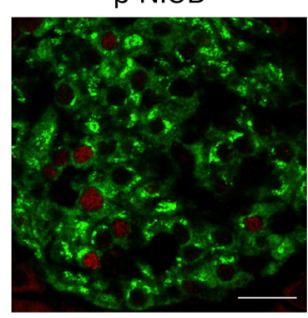

NICD-HA MafA
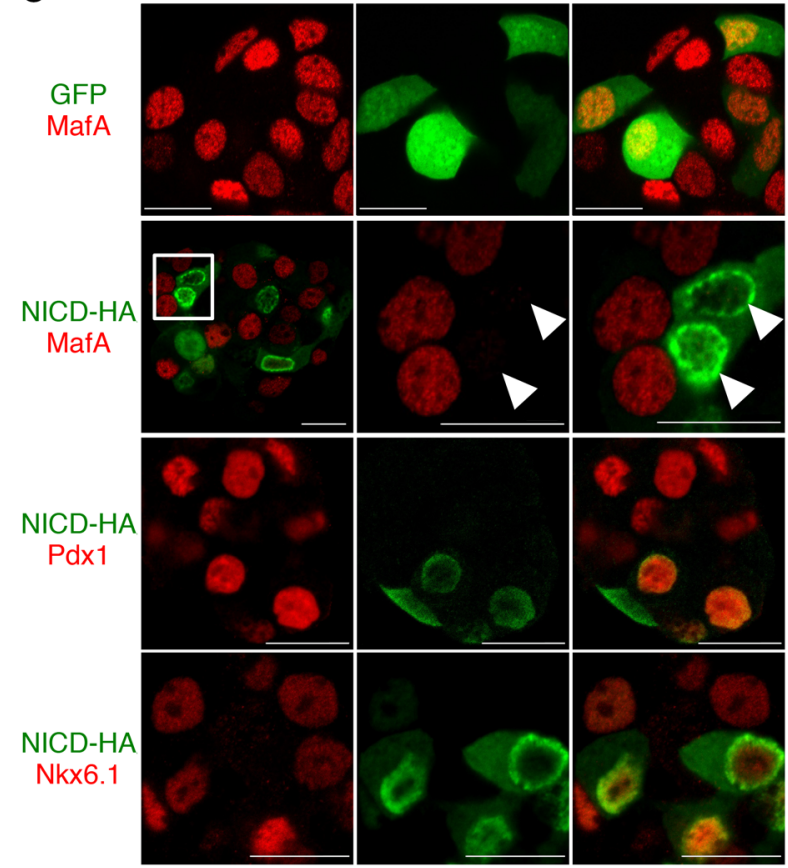

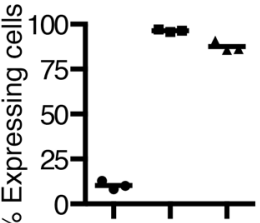

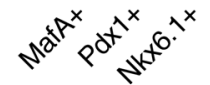

\section{D}

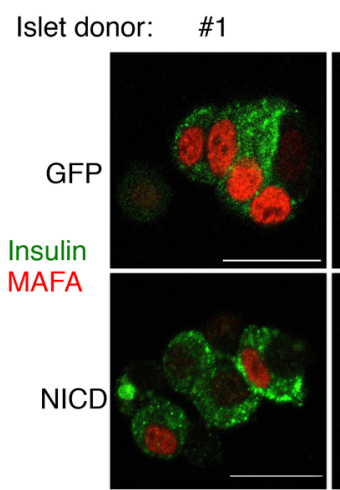

$\mathbf{F}$

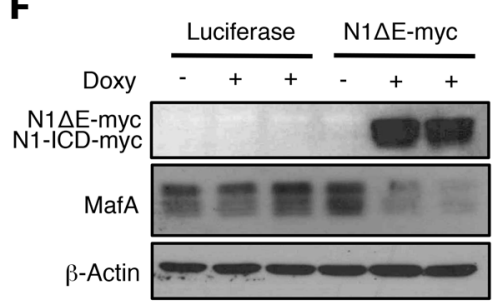

\#2

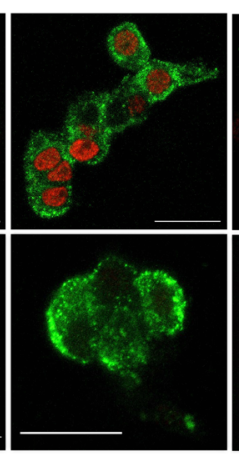

\#3

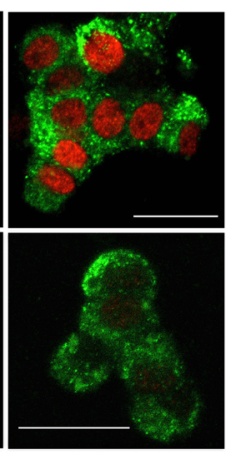

E

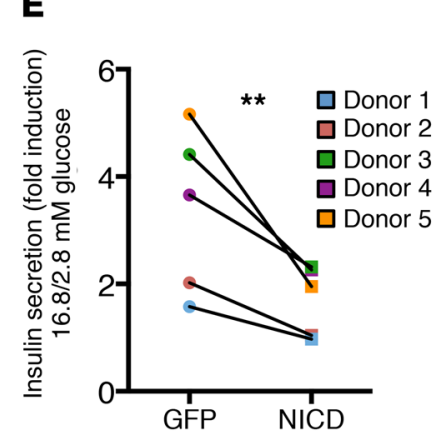

G

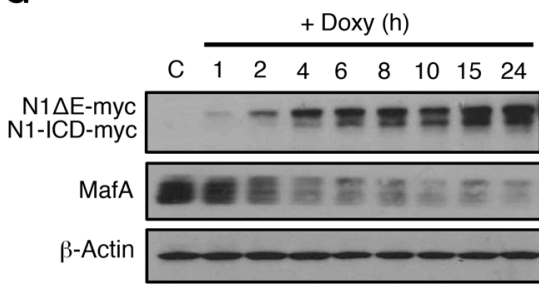

H

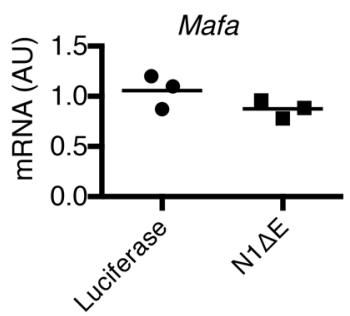

Figure 3. Notch activation leads to loss of MafA in mouse and human $\beta$ cells. (A) Representative images of pancreatic sections from $\beta$-NICD and Crecontrol mice, stained with antibodies directed against insulin and MafA ( $n=6$ mice/group, quantification shown in Supplemental Figure 3C). (B) Western blots in islets isolated from $\beta$-NICD and Cre- control mice. (C) Representative images of immunofluorescence of fixed MIN6-rTTA3 cells transfected with doxycycline-inducible NICD-HA (or GFP control) after stimulation with doxycycline for 24 hours, with quantitation of percentage of NICD+ cells with detectable MafA, Pdx1, or Nkx6.1 ( $n=3$ biologic replicates). Boxed region in second row is shown amplified; arrowheads indicate Notch ${ }^{+}$cells with absent MafA (D) MafA and insulin staining in dispersed human $\beta$ cells transduced with lentivirus expressing GFP or NICD. Representative images from 3 individual donors selected out of a total sample size of 6 donors. (E) GSIS in islets from healthy human donors transduced with lentivirus expressing GFP or NICD. Results are expressed as fold increase in insulin secretion $\left(16.8 \mathrm{mM}\right.$ glucose $/ 2.8 \mathrm{mM}$ glucose) and reflect data from 5 individual donors. ${ }^{* *} P<0.01$, ratio paired $t$ test. (F) Western blots from MIN6-rTTA3 cells with stable integration of doxycycline-dependent N1 $\Delta \mathrm{E}$-myc (or luciferase control) after stimulation with doxycycline for 24 hours. Doxy, doxycycline. (G) Western blots from MIN6-rTTA3 cells with stable integration of doxycycline-dependent N1 $\Delta \mathrm{E}-\mathrm{myc}$ after stimulation with doxycycline for the indicated amount of time. (H) Mafa expression in MIN6-rTTA3 cells with stable integration of doxycyclinedependent N1 $\Delta \mathrm{E}$-myc (or luciferase control) after stimulation with doxycycline for 24 hours ( $n=3$ biologic replicates). Scale bars: $20 \mu \mathrm{m}$. All data are shown with group means. 
A

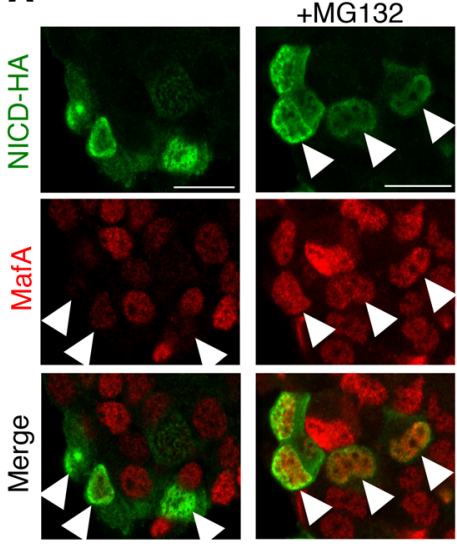

D

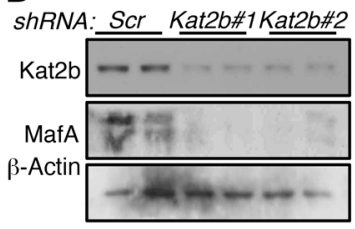

$\mathbf{E}$
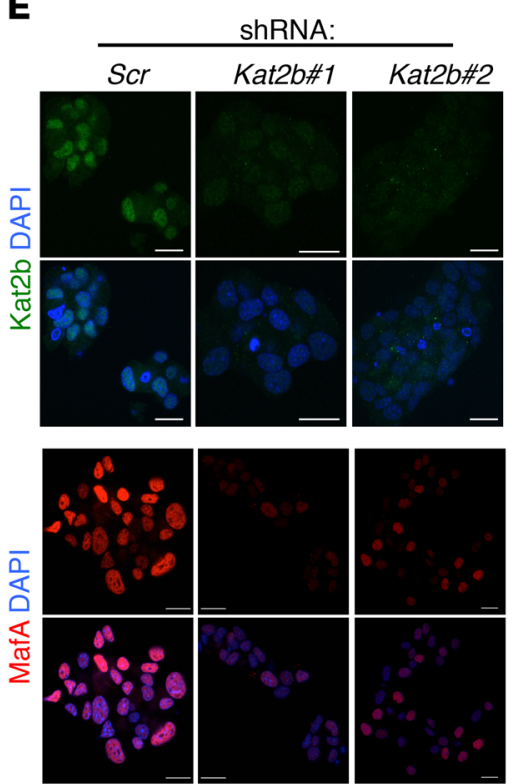

B
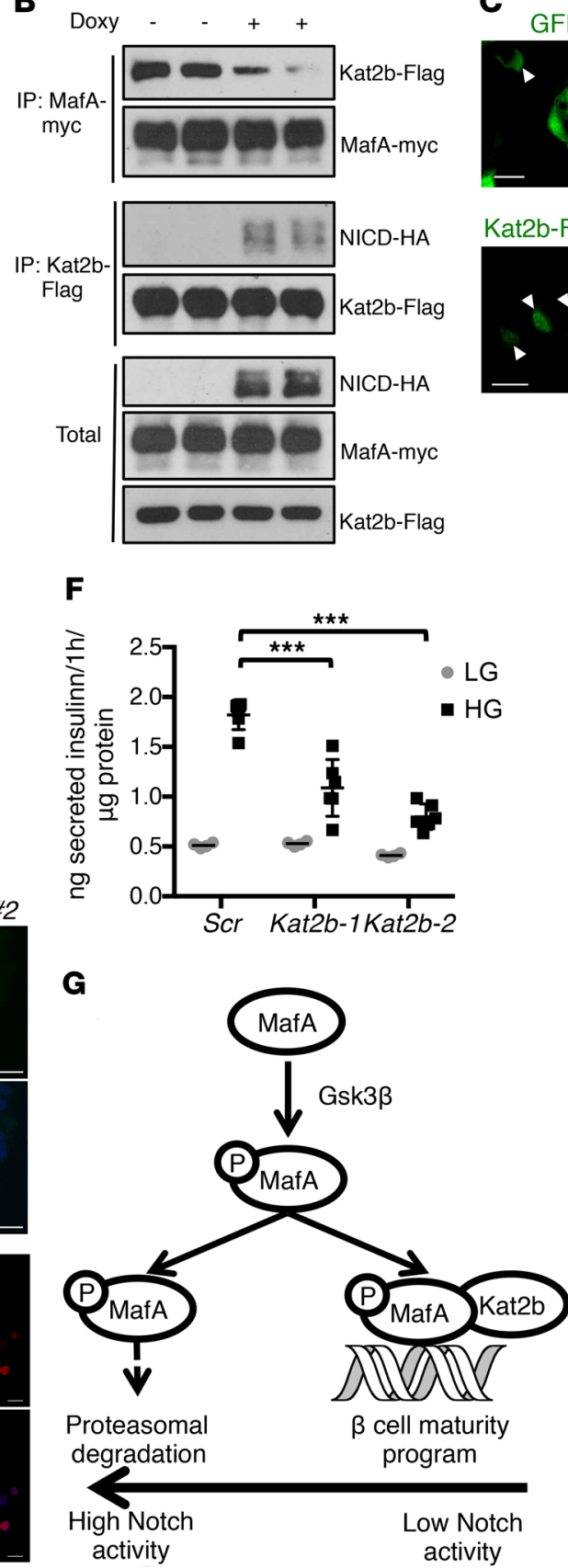

C
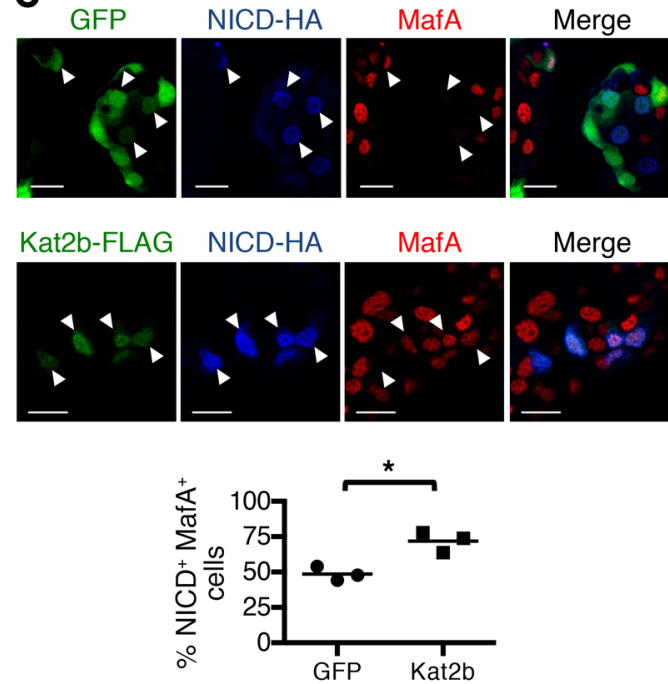

Figure 4. Notch causes MafA degradation by preventing its association with Kat2b. (A) Representative immunofluorescence images of fixed MIN6 cells transiently transfected with NICD-HA, with or without MC132 ( $n=4$ independent experiments). (B) Western blots from immunoprecipitates derived from 293T cells with stable integration of the rTTA3 transcriptional transactivator, transfected with MafA-myc, Kat2b-Flag, and Tet-On NICD-HA, with or without stimulation with doxycycline for 4 hours. (C) Representative images of immunofluorescence of fixed MIN6-rTTA3 cotransfected with Tet-On NICD-HA and GFP or Kat2b-Flag after stimulation with doxycycline for 8 hours, and quantitation of percentages of NICD+CFP+ or NICD+Kat2b+ (arrowheads) cells with detectable MafA ( $n=3$ independent experiments, $\sim 250$ cells analyzed per sample). ${ }^{*} P<0.05$, 2-tailed $t$ test. (D) Western blots from MIN6 cells transduced with lentivirus encoding shRNA targeting Kat2b (2 different sequences: Kat2b\#1 and Kat2b\#2) or scrambled control (Scr). (E) Immunofluorescence images of fixed MIN6 cells transduced with lentivirus encoding shRNA targeting Kat $2 b$ or scrambled shRNA (Scr). Representative images from 2 independent experiments. (F) GSIS from MIN6 cells transduced with lentivirus encoding shRNA against Kat2b or scrambled control in medium containing low $(1 \mathrm{mM}, \mathrm{LG})$ or high $(25 \mathrm{mM}, \mathrm{HC})$ glucose. Results from a representative experiment with 6 replicates per condition. Experiment was performed 3 times. ${ }^{* * *} P<0.001$, 1-way ANOVA and Dunnett's multiple comparisons post hoc test. (G) Model of Notch regulation of MafA-Kat2b interaction and MafA proteasomal degradation. Scale bars: $20 \mu \mathrm{m}$. All data are shown with group means. 
A

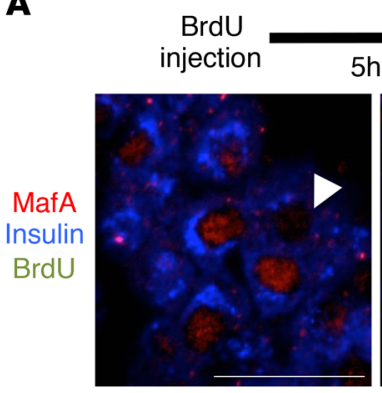
$5 \mathrm{~h}$

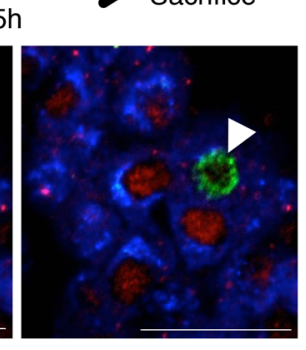

C
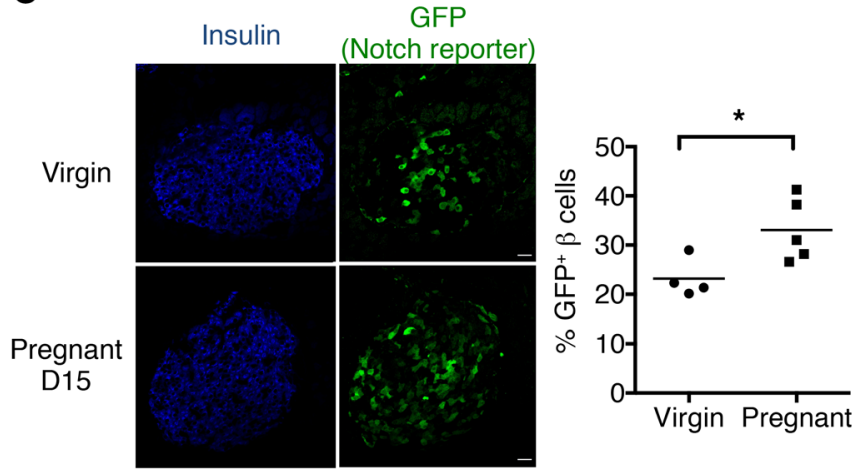

B

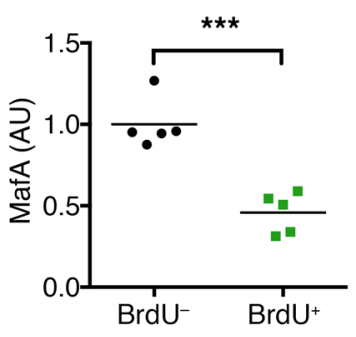

Pdx1

Insulin

BrdU
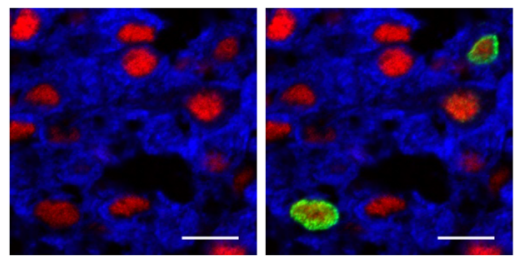

Neurod1
Insulin

BrdU
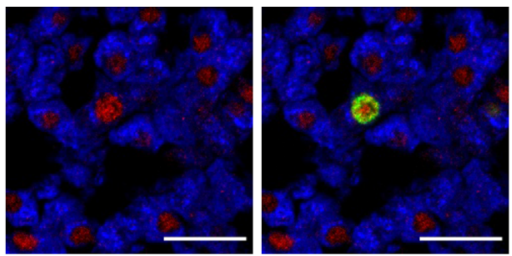

Ucn3

Insulin

BrdU
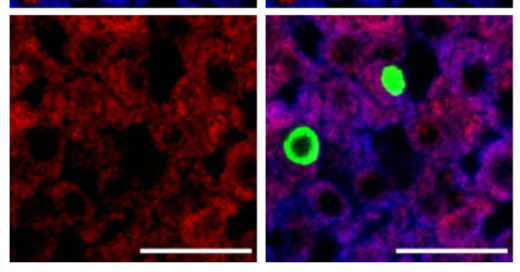

D
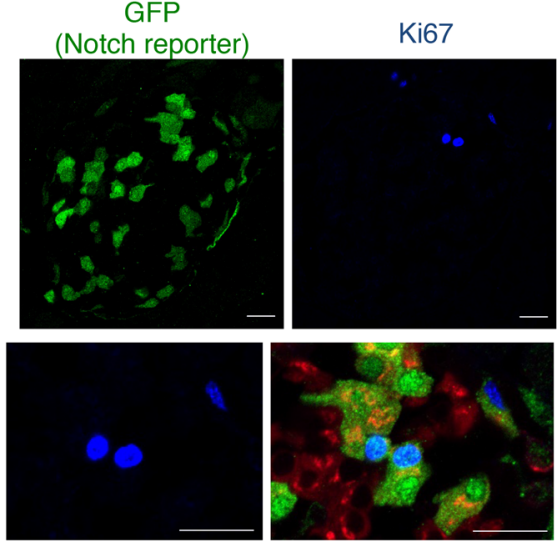

Ki67
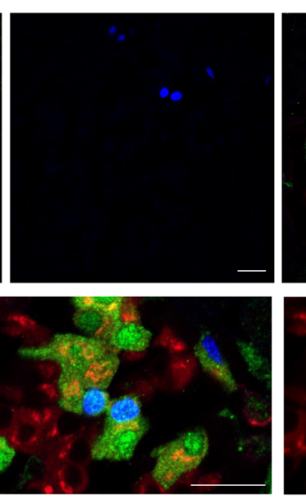

Insulin
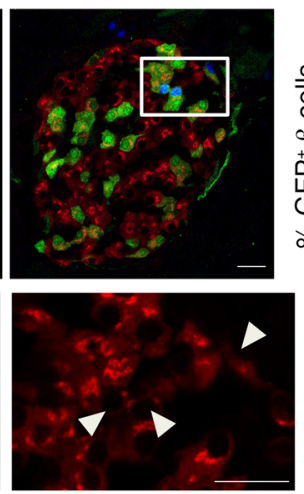

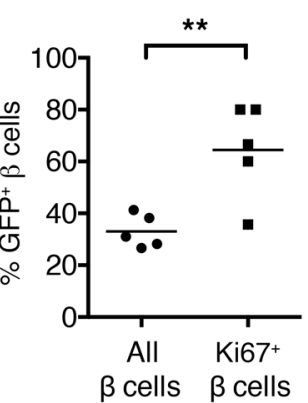

E

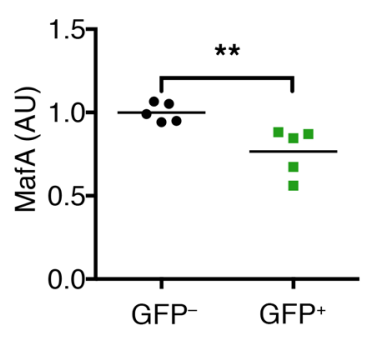

Figure 5. Notch activation and reduced maturity in proliferating $\boldsymbol{\beta}$ cells. (A) Representative images of pancreatic sections from day 15 (D15) pregnant (D15) WT mice and quantitation expressed as average MafA fluorescence intensity of BrdU ${ }^{+}$and $\mathrm{BrdU}^{-}$populations ( $n=5$ mice/group). Individual cells are plotted in Supplemental Figure 6A. (B) Representative images of pancreatic sections from pregnant (D15) mice stained for BrdU as well as antibodies directed against insulin and Pdx1, Neurod1, or Ucn3 ( $n=5$ mice/group). (C) Representative images of pancreatic sections from virgin and pregnant (D15) TNR mice, with quantitation of percentage of GFP+ $\beta$ cells ( $n=4-5$ mice/group). (D) Representative images of pancreatic sections from pregnant (D15) TNR mice showing Ki67, insulin, and GFP reporter staining (top), with increased magnification of the boxed region (bottom). Arrows indicate Ki67 $\beta$ cells. Quantitation of percentage of GFP+ $\beta$ cells or percentage of GFP+Ki67+ $\beta$ cells ( $n=5$ mice/group; overall count of 3995 total $\beta$ cells and 188 Ki67 $\beta$ cells). (E) Quantitation of average MafA fluorescence intensity in GFP- and GFP+ $\beta$ cells from chow-fed TNR mice, normalized to average value in GFP- cells ( $n=5$ mice). Approximately 1000 cells quantified per pancreas. Scale bars: $20 \mu \mathrm{m}$. All data are shown with group means. ${ }^{*} P<0.05 ;{ }^{* *} P<0.01 ;{ }^{* *} P<0.001,2$-tailed $t$ test.

( $\beta$-DNMAML) mice, which showed decreased $\beta$ cell proliferation at P14 (Figure 6F). To establish whether Notch regulation of $\beta$ cell proliferation was cell autonomous, we isolated islets from MIP- $\beta$-NICD and MIP- $\beta$-DNMAML mice, then treated them with 4-OHT to induce recombination. Consistent with in vivo results, forced Notch activation increased glucose-induced proliferation, while genetic blockade of $\beta$ cell Notch activation reduced proliferation (Figure 6G and Supplemental Figure 7D). Importantly, we observed unchanged proliferation capacity when cells were cultured in low glucose, where proliferation rates remained low (Supplemental Figure 7E). Similarly, Notch gain of function enhanced proliferation during pregnancy (Figure 6H), whereas pregnancy-induced $\beta$ cell proliferation was diminished in Notch loss-of-function mice (Figure 6I). We conclude from these results that endogenous Notch activation augments $\beta$ cell proliferation in times of need, but simultaneously suggest that Notch is neither necessary nor sufficient to force proliferation-incompetent (i.e., aging, low-glucose growth conditions) $\beta$ cells to divide.

$\beta$ Cell Notch inhibition improves obesity-induced glucose intolerance. We next circled back to our original observations and 
A

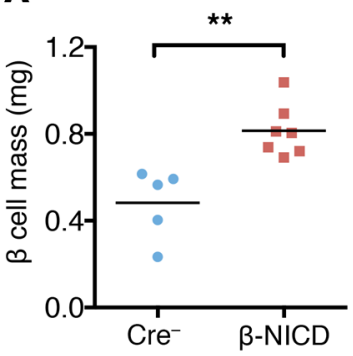

D

Control

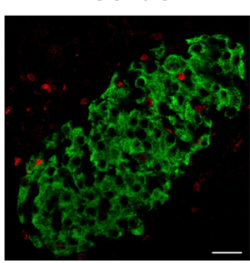

P14 pancreas

Insulin Ki67

$\mathbf{F}$

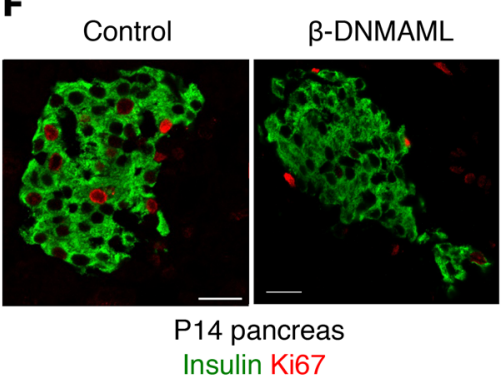

H

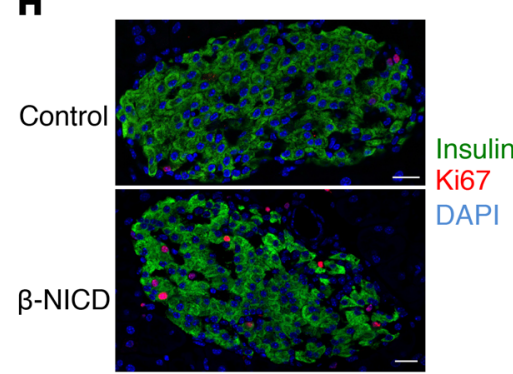

B

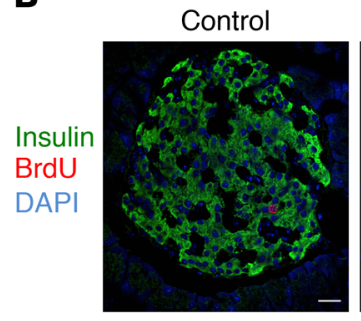

$\beta-N I C D$

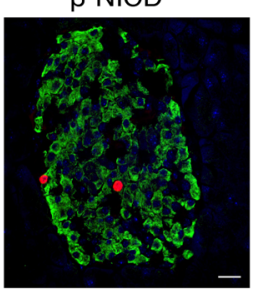

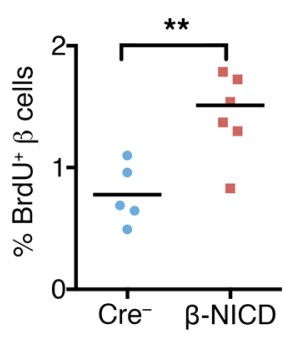

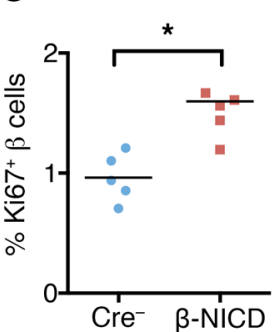

E
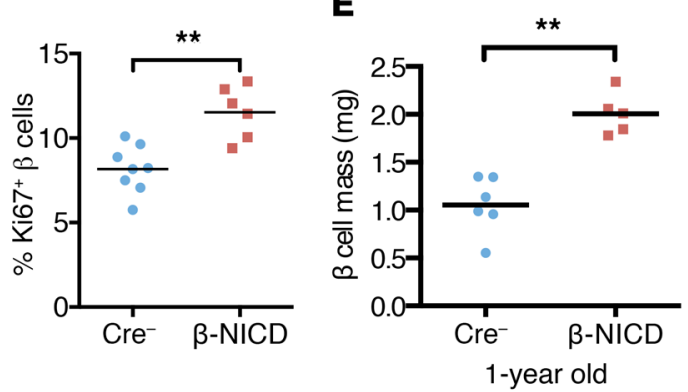

G
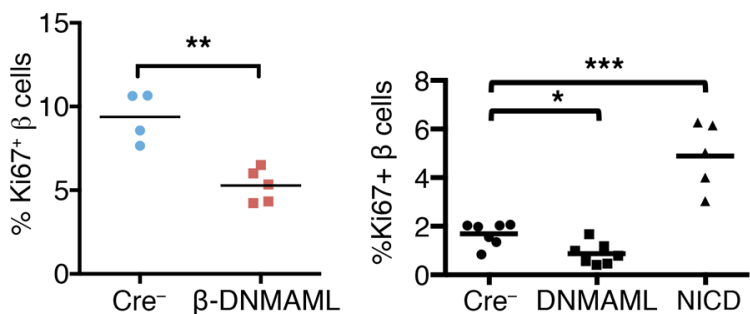
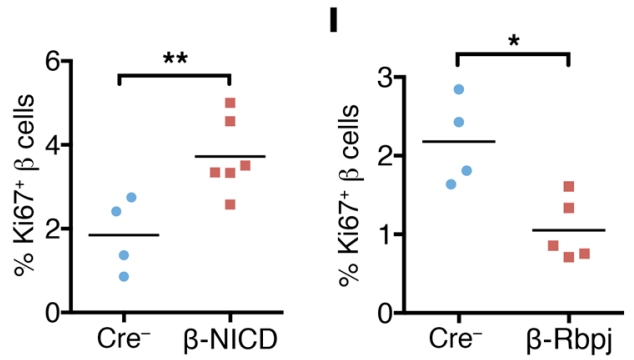

Figure 6. Notch activation increases $\beta$ cell proliferative capacity. (A) Quantitation of $\beta$ cell mass in adult $\beta$-NICD and Cre- control mice ( $n=5-7$ mice/group). ${ }^{* *} P<0.01$, 2-tailed $t$ test. (B) Representative images and quantitation of BrdU+ $\beta$ cells in pancreatic sections from adult $\beta$-NICD and Cre- control mice ( $n=5$ mice/group). ${ }^{* *} P<0.01,2$-tailed $t$ test. (C) Quantitation of Ki67 $\beta$ cells in pancreatic sections from adult $\beta$-NICD and Cre ${ }^{-}$control mice ( $n=5$ mice/group). ${ }^{*} P<0.05$, 2-tailed $t$ test. (D) Representative images and quantitation of Ki67+ $\beta$ cells in P14 pancreas in $\beta$-NICD and Cre- control mice ( $n=6-8$ mice/group). ${ }^{* *} P<0.01$, 2-tailed $t$ test. (E) Quantitation of $\beta$ cell mass in 1-year old $\beta$-NICD and Cre- mice ( $n=5-6$ mice/group). ${ }^{* *} P<0.01,2$-tailed $t$ test. (F) Representative images and quantitation of Ki67 $\beta$ cells in P14 pancreas in $\beta$-DNMAML and Cre- control mice $\left(n=4-5\right.$ mice/group). ${ }^{* *} P<0.01$, 2-tailed $t$ test. (C)

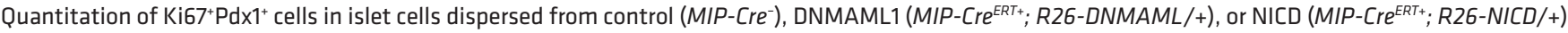
mice, grown in full medium containing $22 \mathrm{mM}$ glucose and $1 \mu \mathrm{M} 4-\mathrm{OHT}$ for 4 days $\left(n=5-7\right.$ mice/group). ${ }^{*} P<0.05$; ${ }^{* *} P<0.001,1$-way ANOVA and Dunnett's multiple comparisons post hoc test. Representative images are shown in Supplemental Figure 6D. (H) Representative images and quantitation of Ki67 $\beta$ cells in pancreatic sections from pregnant (D15) $\beta$-NICD and Cre ${ }^{-}$control females ( $n=4-6$ mice/group). ${ }^{* *} P<0.01,2$-tailed $t$ test. (I) Quantitation of Ki67 $\beta$ cells in pancreatic sections of pregnant (D15) $\beta$-Rbpj and Cre- control females ( $n=4-5$ mice/group). ${ }^{*} P<0.05,2$-tailed $t$ test. Scale bars: $20 \mu \mathrm{m}$. All data are shown with group means.

hypothesized that blocking Notch signaling in $\beta$ cells may increase MafA stability and improve obesity-induced glucose intolerance. Indeed, expression of DNMAML reversed loss of MafA seen in primary $\beta$ cells challenged with high glucose and palmitate (Figure
7A). Consistently, DNMAML increased the half-life of exogenous MafA in cycloheximide-treated MIN6 cells (Figure 7B).

To determine whether $\beta$ cell-specific Notch loss of function could reverse loss of MafA in HFD-fed mice (Supplemental Fig- 
A

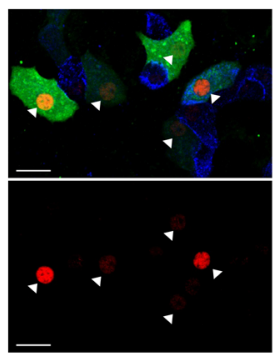

Insulin DAPI DNMAML-GFP MafA

C

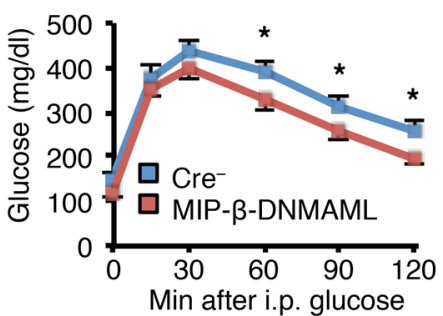

$\mathbf{F}$

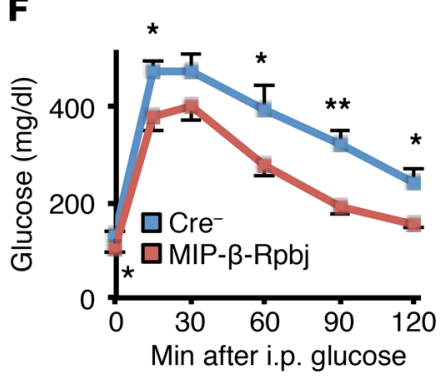

B
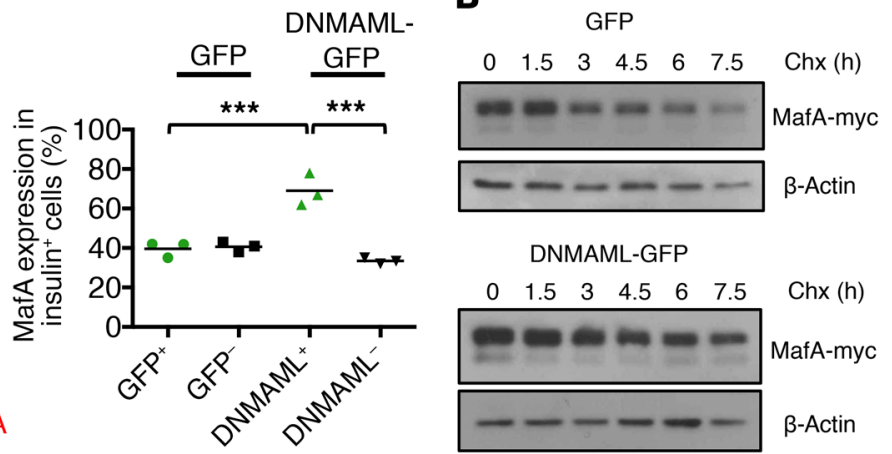

D $\mathrm{Cre}^{-}$
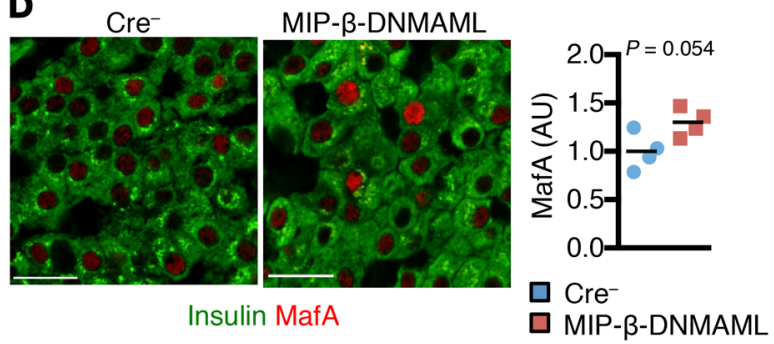

G

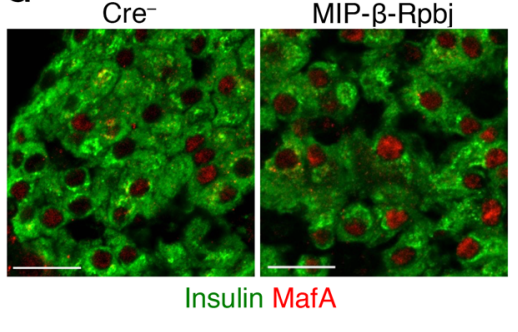

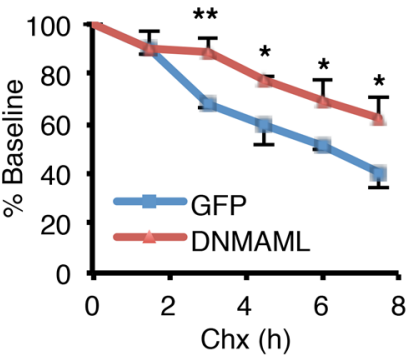

E

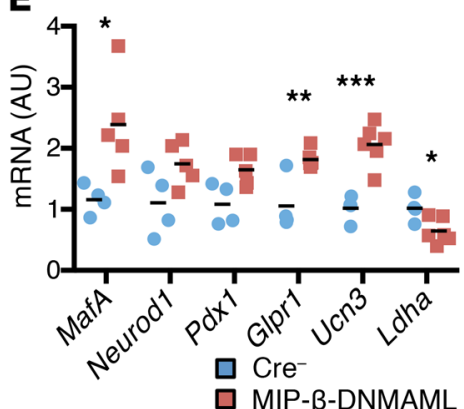

H

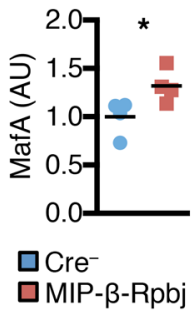

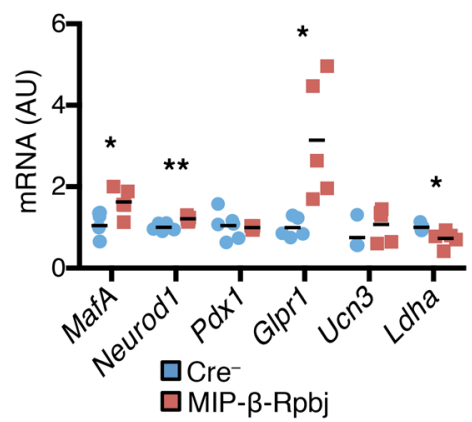

Figure 7. Notch loss of function stabilizes $\boldsymbol{\beta}$ cell MafA and improves glucose tolerance. (A) Representative image of islet cells dispersed from WT mice transduced with lentivirus expressing DNMAML-GFP (or GFP control) and exposed to $100 \mathrm{nM}$ palmitate and 25 mM glucose for 72 hours. Arrows indicate DNMAML ${ }^{+}$cells. Quantitation shows insulin MafA $^{+}$islet cells in transduced vs. nontransduced cells $\left(n=3\right.$ independent experiments). ${ }^{* * *} P<0.001,1$-way ANOVA and Dunnett's multiple comparisons post hoc test. (B) Western blots and quantitation of residual MafA-Myc signal expressed as percentage of baseline from MIN6 cells with stable integration of DNMAML-GFP (or GFP control) transiently transfected with MafA-myc, then treated with $10 \mu \mathrm{g} / \mathrm{ml}$ cycloheximide (Chx) for the indicated times ( $n=3$ independent experiments). ${ }^{*} P<0.05 ;{ }^{*} P<0.01,2$-tailed $t$ test. (C) GTT in TAM-treated, HFD-fed MIP- $\beta$-DNMAML and $\mathrm{Cre}^{-}$control mice ( $n=9-11$ mice/group) per experimental protocol shown in Supplemental Figure $8 \mathrm{C} .{ }^{*} P<0.05,2$-tailed $t$ test. (D) Representative images and quantitation of MafA fluorescence intensity in pancreatic sections from HFD-fed MIP- $\beta$-DNMAML and Cre ${ }^{-}$control mice $(n=4$ mice/group). $P=0.054$, 2-tailed $t$ test. (E) Gene expression in islets isolated from HFD-fed MIP- $\beta$-DNMAML and Cre- control mice $\left(n=4-5\right.$ mice/group). ${ }^{*} P<0.05 ;{ }^{* *} P<0.01 ;{ }^{* * *} P$ $<0.001$, 2-tailed $t$ test. (F) GTT in HFD-fed MIP- $\beta$-Rbpj and Cre- control mice ( $n=9$ mice/group). ${ }^{*} P<0.05$; ${ }^{* *} P<0.01$, 2-tailed $t$ test. (G) Representative images and quantitation of MafA fluorescence intensity in pancreatic sections from HFD-fed MIP- $\beta$-Rbpj and Cre- control mice ( $n=4$ mice/group). ${ }^{*} P<0.05$, 2-tailed $t$ test. (H) Gene expression in islets isolated from HFD-fed MIP- $\beta$-Rbpj and Cre- control mice ( $n=5$-6 mice/group). ${ }^{*} P<0.05$; ${ }^{* *} P<0.01,2$-tailed $t$ test. Scale bars: $20 \mu \mathrm{m}$. All data are shown with group means \pm SEM.

ure 8, A and B), we utilized MIP- $\beta$-DNMAML mice, but delayed TAM-induced recombination and HFD feeding until midadulthood (Supplemental Figure 8C), both to bypass the $\beta$ cell proliferation differences attributable to Notch in the early postnatal period and to minimize the proliferative effect of the human growth hormone $(\mathrm{hGH})$ minigene in the MIP-Cre ${ }^{\text {ERT }}$ transgene $(26,27)$. We confirmed the utility of this strategy, as WT and MIPCre ${ }^{\mathrm{ERT}}$ mice (without floxed alleles) showed similar glucose tolerance, body weight, $\beta$ cell proliferation, and $\beta$ cell mass when HFD feeding was initiated at 24 weeks of life (Supplemental Figure 8, $D-G)$, in contrast with MIP-Cre ${ }^{\text {ERT }}$ mice fed HFD when $\beta$ cells were more proliferative (26). With this experimental paradigm, MIP- $\beta$ DNMAML mice demonstrated improved glucose tolerance (Figure 7C). As predicted, islets from these mice showed lower Notch activity (Supplemental Figure $8 \mathrm{H}$ ), but also indications of greater $\beta$ cell maturity (Figure 7, D and E).

To ensure reproducibility, we created a second, inducible Notch loss-of-function mouse model, MIP- $\beta$-Rbpj mice, which 
showed normal glucose tolerance when fed a chow diet (Supplemental Figure 8I), but improved glucose tolerance as compared with $\mathrm{Cre}^{-}$controls when challenged with HFD feeding (Figure $7 F)$. Consistent with data from MIP- $\beta$-DNMAML mice, islets from MIP- $\beta$-Rbpj mice had decreased Notch activation (Supplemental Figure 8J), with a parallel increase in MafA levels, leading to a promaturity $\beta$ cell expression profile (Figure 7, G and H). Finally, as predicted, given the delay in HFD-induced insulin resistance until $\beta$ cells were less proliferative, both Notch loss-of-function models showed $\beta$ cell mass and $\beta$ cell proliferation that were the same as those of $\mathrm{Cre}^{-}$controls and therefore had identical fasted serum insulin levels (Supplemental Figure 8, I-K, and not shown). These data indicate that Notch inhibition stabilizes MafA, leading to increased $\beta$ cell maturity and improved glucose tolerance in the face of obesity-induced metabolic challenge.

\section{Discussion}

Reactivation of "embryonic" signaling pathways can occur in environmentally stressed $\beta$ cells (3). For instance, increased Notch signaling has been shown in hypoxia (28) or when islets are compromised in the setting of pericyte ablation (29). Since these same stressors also lead to $\beta$ cell dysfunction, cause and effect cannot be disentangled, but here we show that unrestrained Notch activation is detrimental to $\beta$ cell maturity and leads to disrupted GSIS in isolated mouse and human islets as well as impaired glucose tolerance in vivo in conditions associated with increased systemic demand for insulin. Reciprocally, genetic inhibition of Notch activation maintains $\beta$ cell function when mice are challenged by HFD feeding independently of Notch's well-established role in pancreatic endocrine development $(6,7,30)$.

Our data also indicate that constitutive Notch activation cannot induce dedifferentiation, unlike forced activation of Hedgehog signaling (31), nor is Notch activation sufficient to trigger $\beta$ cell replication, but rather, it leads to a higher proliferative rate under conditions associated with $\beta$ cell division. These data suggest that Notch activation pushes stressed $\beta$ cells further away from the status quo, but whether the positive effect of Notch signaling on $\beta$ cell proliferation is a direct consequence of Notch activation, a byproduct of loss of $\beta$ cell maturity, or both is not yet known. These findings do, however, support the idea of $\beta$ cell function being opposed to $\beta$ cell proliferation (32) and also support a model in which the proliferating population of $\beta$ cells is both Notch active and functionally immature, with a readout of destabilized MafA. These data are consistent with in vitro work showing Notch activation in proliferating human $\beta$ cells $(33,34)$ as well as studies on proliferating $\beta$ cells in vivo (23), which showed repression of the Pdx1/Neurod1/MafA network, but not gene expression of these canonical $\beta$ cell transcription factors or loss of other maturity markers, such as Ucn3 (35). Of these, MafA has been consistently shown to be essential for $\beta$ cell function and survival in the adult pancreas (36-38). Various circumstantial data suggest that partial loss of MafA activity may represent commitment to $\beta$ cell division: (a) forced MafA expression in pancreatic progenitors increased expression of cell cycle inhibitors and blunted cell proliferation (39); (b) MafA only becomes essential for $\beta$ cell function after weaning (36); and(c) MafA expression is low during the postnatal proliferative wave (40) and in hyperproliferative human insulino- mas with altered MENIN expression (41). However, total ablation of MafA in whole body or pancreas-specific knockout models does not result in hyperproliferative $\beta$ cells - on the contrary, loss of $\beta$ cell mass was reported $(36,38)$. One possible explanation that loss of MafA may lead to compensatory MafB expression, reported to mediate $\beta$ cell proliferative adaptation to pregnancy $(42,43)$ and $\beta$ cell maturation $(44,45)-$ did not hold true in Notch-active $\beta$ cells. This may indicate that dynamic regulation of MafA activity is required for normal regulation of $\beta$ cell maturity and proliferation, perhaps by Notch effects on Kat2b-MafA interaction. Thus, our data are broadly consistent with the protective role of MafA for $\beta$ cell adaption to stress (46-48), but further studies are necessary to test MafA dependence on Notch's various effects on $\beta$ cell biology as well as to determine Kat2b interaction partners in $\beta$ cells in various pathophysiologic states.

We observe heterogeneous Notch activation in $\beta$ cells, consistent with differential proliferative capability of distinct $\beta$ cell populations $(49,50)$; in fact, the proliferative "bottom $\beta$ cell population" identified by Rui and colleagues shows increased Hes1 expression (50). Notch activation is at minimum a readout of this $\beta$ cell heterogeneity, but likely accounts for variability in cell-to-cell MafA and resultant propensity to proliferate. Although we have not yet identified the source of the Notch signal, our preliminary data point to $\beta$ cell-specific upregulation of specific Notch ligands of the Delta-like and Jagged families in response to hyperglycemia, which suggests the possibility of stressed $\beta$ cells sending out a call for proliferation. But our data cannot exclude other endocrine or non-endocrine signal-sending cells; similarly, as our Notch loss-of-function mouse models focused on common downstream signaling components (MAML, Rbpj), future studies will be necessary to identify the specific Notch receptor activated in $\beta$ cells.

In sum, we find active Notch signaling in the "normal" $\beta$ cell, but with 2 disparate outcomes: in young mice, Notch activation represents an adaptive mechanism to increase $\beta$ cell mass induced by insulin resistance by permissive action on proliferation, but in aged mice with lower proliferative capability (51) or with chronic insults, such as obesity, Notch activation locks $\beta$ cells into an immature and dysfunctional state - a maladaptive compensatory response. Thus, Notch loss of function enhances $\beta$ cell maturity and improves glucose tolerance. These data parallel findings in liver (9) and other insulin-sensitive tissues $(52,53)$, where similar Notch "reactivation" is causal to insulin resistance $(10,11,54-56)$. As Notch is therapeutically accessible (57), with small molecules and biologics in the pipeline for cancer (58), these results provide the groundwork to repurpose these agents to address the dual pathologies - insulin resistance and deficient insulin secretion - that characterize T2D.

\section{Methods}

Animals. RIP-Cre: Tg(Ins2-cre)23Herr (16), MIP-Cre ${ }^{E R T}$ : B6.Cg-Tg(Ins1-cre) ERT)1Lphi/J (17), R26-NICD: Gt(ROSA)26Sor rm1(Notchl)Dam (7), R26-DN-

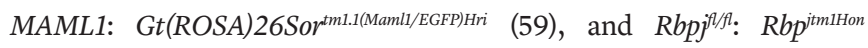
(6) mouse lines were maintained on a C57BL/6J background. TNR: $\mathrm{Tg}(\mathrm{Cp}$-EGFP)25Gaia/ReyaJ mice were maintained in homozygosity to avoid epigenetic silencing on a C57BL/6J and SJL/J mixed background (12). H2B-Venus notch reporter: $\mathrm{Tg}$ (Cp-HIST1H2BB/Venus) $47 \mathrm{Hadj}$ mice were maintained in homozygosity on a FVB background (13). Mice were 
weaned and maintained on standard chow (Purina Mills, catalog 5053) or HFD (Harlan Laboratories, TD.06414) as stated. Adult mice (8-12 weeks old) were used for experiments, unless stated otherwise. No differences in body weight or glucose tolerance were found between WT and RIP-Cre or MIP-Cre ${ }^{E R T}$ mice (not shown). Male mice were used for all experiments described, with the exception of experiments involving pregnancy, and ex vivo GSIS, where islets from mice and female mice were used without distinction.

Antibodies and chemicals. All antibodies used can be found in Supplemental Table 1. STZ, TAM, 4-OHT, doxycycline hyclate, MG132, cycloheximide, $\mathrm{BrdU}$, and $\mathrm{LiCl}$ were obtained from MilliporeSigma. CHIR 99021 was obtained from Tocris Bioscience.

STZ and TAM treatment. STZ was dissolved in cold citrate buffer, $0.1 \mathrm{M} \mathrm{pH} 4.5$, and injected within 10 to 15 minutes of preparation, once daily at a dose of $50 \mathrm{mg} \mathrm{STZ/kg} \mathrm{body} \mathrm{weight} \mathrm{for} 5$ consecutive days. Mice were sacrificed 18 days after last STZ injection, with fed glucose levels of $450-520 \mathrm{mg} / \mathrm{dl}$. TAM was dissolved in corn oil (MilliporeSigma) and sterile filtered; both $\mathrm{Cre}^{-}$controls and $\mathrm{CreERT}^{+}$mice were treated with $100 \mathrm{mg}$ TAM/kg body weight once daily for 3 consecutive days.

Islet isolation. Islets were routinely isolated by collagenase P (Roche Applied Science) digestion of whole pancreas (except for electron microscopy experiments), followed by Histopaque density-gradient centrifugation, as described (60). When required, islets were maintained ex vivo in $5.5 \mathrm{mM}$ glucose RPMI 1640 supplemented with 10\% FBS and 1\% penicillin-streptomycin. For islet dispersion experiments, isolated islets were incubated overnight and dissociated with Accutase (Corning). Dispersed cells were plated onto glass coverslips and cultured on islet medium.

Human islet studies. Human islets from 6 nondiabetic donors (ages 25-57 years old, BMI $23.6-33.5 \mathrm{~kg} / \mathrm{m}^{2}$ ) were obtained through the Integrated Islet Distribution Program (IIDP) via NIH-approved centers. Islets were cultured in PIM(S) media supplemented with $5 \%$ human serum (Prodo Labs).

Glucose tolerance tests and GSIS assays. MIN6 cells were incubated in $1 \mathrm{mM}$ glucose Krebs-Ringer-HEPES (KRH) buffer (129 mM NaCl, 5 $\mathrm{mM} \mathrm{NaHCO}_{3}, 4.8 \mathrm{mM} \mathrm{KCl}, 1.2 \mathrm{mM} \mathrm{KH}_{2} \mathrm{PO}_{4}, 1.2 \mathrm{mM} \mathrm{MgSO}_{4}, 1.2 \mathrm{mM}$ $\mathrm{MgSO}_{4}, 2.5 \mathrm{mM} \mathrm{CaCl}_{2}, 10 \mathrm{mM}$ HEPES $\mathrm{pH}$ 7.4, 0.1\% BSA) for 1 hour, then transferred to $20 \mathrm{mM}$ glucose for 1 hour. For islet GSIS, after overnight culture in $5.5 \mathrm{mM}$ glucose RPMI, groups of 6 similar size islets were incubated for 1 hour in $2.8 \mathrm{mM}$ glucose KRH buffer, subjected to $2.8 \mathrm{mM}$ and $16.8 \mathrm{mM}$ glucose stimulation, and supernantants collected. For total insulin content, islets were transferred to high-salt buffer (2.15 $\mathrm{M} \mathrm{NaCl}, 10 \mathrm{mM} \mathrm{NaH}_{2} \mathrm{PO}_{4}, 40 \mathrm{mM} \mathrm{Na}_{2} \mathrm{HPO}_{4}, 1.8 \mathrm{mM} \mathrm{EDTA} \mathrm{pH} \mathrm{7.4)} \mathrm{and}$ sonicated for 2 minutes in a Bioruptor sonicator (Diagenode). Insulin in supernatant was detected by ELISA (Mercodia). Total islet DNA content was determined by quantitative PCR (qPCR) using a standard curve of defined DNA values. Glucose tolerance tests (GTTs) were performed as previously described (10).

Cell lines and lentivirus. Culture of insulinoma-derived MIN6 cells and lentivirus generation with the use of 293T cells were performed as described (61). For generation of a MIN6 cell line expressing a transcriptional transactivator (MIN6-rTTA3), cells were transduced with lentivirus obtained with the pLenti CMV rtTA3 Blast vector (Addgene, catalog 26429) and a blasticidin ( $5 \mu \mathrm{g} / \mathrm{ml})$ selection. Vectors for shRNAmediated knockdown of Mafa or Kat $2 b$ were generated by cloning targeting sequences (Supplemental Table 2) into pLKO.1-TRC cloning vector (Addgene, catalog 10879). To generate stable MIN6-rTTA3 cells for doxycycline-mediated expression of $\mathrm{N} 1 \Delta \mathrm{E}-\mathrm{myc}$ or luciferase, $\mathrm{N} 1 \Delta \mathrm{E}$
cDNA was cloned from pCS2 Notch1 $\triangle$ EMV-6MT (Addgene, catalog 41737) into pLVX-Tight-Puro vector (Clontech) with pLVX-Tight-Luc (Clontech) used as a negative control. Lentiviral particles generated from these vectors were transduced into MIN6-rTTA3 cells, which were selected with $1 \mu \mathrm{g} / \mathrm{ml}$ puromycin (MilliporeSigma). MIN6 cells stably expressing DNMAML-GFP or GFP control were generated after lentivirus infection and FACS sorting. All vectors used in this study can be found in Supplemental Table 3.

Lentivirus-mediated transduction. Lentivirus was generated in $293 \mathrm{~T}$ cells, as previously described (61). Titration was performed by qPCR. Intact or dispersed islets were transduced with 10 transducing units (TU)/cell, assuming that an average islet contains 1000 cells, in complete medium enriched with $8 \mu \mathrm{g} / \mathrm{ml}$ sequabrene (MilliporeSigma). Imaging and GSIS experiments in human islets were performed 96 hours after lentivirus infection.

Western blotting and PCR. Lysates were resolved by SDS-PAGE, followed by Western blot and visualization using the ECL Western Blotting Detection Kit (GE Healthcare Bio-Sciences). RNA was isolated with TRIzol (Thermo Fisher), cDNA obtained with the HighCapacity cDNA Reverse Transcription Kit (Thermo Fisher), and qPCR performed using Power SYBR Master Mix (Thermo Fisher). Primer sequences used are detailed in Supplemental Table 2.

Immunofluorescence, $\beta$ cell mass determination, BrdU, and TUNEL assay. Tissue was fixed in $4 \%$ paraformaldehyde/PBS at $4^{\circ} \mathrm{C}$ for 2 to 4 hours, followed by overnight incubation in $30 \%$ sucrose/PBS. Tissue was embedded in OCT compound (Sakura) and flash frozen in dry icechilled isopentane. Tissue sections $5 \mu \mathrm{M}$ thick were processed according to standard procedures. For MafA and Hes1 immunostaining, slides were incubated for 20 minutes at $70^{\circ} \mathrm{C}$ with HistoVT One (Nacalai) for antigen retrieval. For BrdU staining, mice were injected intraperitoneally with $100 \mathrm{mg} / \mathrm{kg} \mathrm{BrdU}$ (MilliporeSigma) 5 hours before sacrifice. After standard processing of the tissue, slides were incubated at $37^{\circ} \mathrm{C}$ in $2 \mathrm{~N} \mathrm{HCl}$ for 30 minutes, followed by incubation in $0.1 \mathrm{M}$ sodium borate buffer $\mathrm{pH} 8.5$ for 10 minutes, prior to incubation in primary antibodies, then donkey secondary antibodies (Jackson ImmunoResearch), both diluted in PBS containing 5\% normal donkey serum, 1\% BSA, and 0.1\% Triton X-100. TUNEL assay was performed with the In Situ Cell Death Detection Kit, Fluorescein (Roche Applied Sciences) following the instructions from the manufacturer, and pancreas sections further stained with insulin and DAPI. The $\beta$ cell mass analyses and immunofluorescence in MIN6 cells dispersed or intact islets were performed as previously described (60).

Confocal microscopy. For confocal microscopy assays, Axio Observer Z1 with LSM 710 scanning module was used (Zeiss). Images were obtained with a $\times 40$ Zeiss Plan-Apochromat oil objective. Imaging was performed in a single confocal microscopy session for each experiment. For each session, the photomultiplier voltage settings (below 600V) and laser transmission ( $\leq 2 \%$ ) for each fluorophore were determined to maximize the dynamic range of the signal. Controls were used for each experiment to confirm specific signals. All images were obtained in a $1024 \times 1024$ pixel format. For microscope operation and image gathering, ZEN (Zeiss) software was used.

Quantification of immunofluorescence experiments. Quantitative analysis of fluorescence intensity for nuclear antigens (MafA, Pdx1, or Hes1) was performed with images gathered from pancreas tissue slides. For this, raw multichannel LSM files were processed with Image $(\mathrm{NIH})$, and the channel of interest subjected to background correction using the "rolling ball" algorithm implemented in ImageJ. 
The region of interest (ROI) was then drawn on 20-30 random nuclei per image, while visualizing the insulin channel to select $\beta$ cells. The signal intensity for the ROI of the channel of interest (nuclear staining) was obtained per image and then averaged per pancreas (5-10 images per pancreas). Results shown are the average of 3-6 mice per group. The average pixel intensity values were referenced to the values obtained for the control of each experiment and expressed as AU. For qualitative analysis (Ki67, BrdU, MafA expression in vitro), a threshold was set using the Threshold function of ImageJ, and positive and negative cells were counted. Images were processed with ZEN2; the only manipulation performed was linear contrast stretching to attain the optimal dynamic signal range for each set of images. Representative pictures shown for these experiments have quantified values approximated to the final average.

Transmission electron microscopy. Islets were isolated with $0.1 \mathrm{mg} /$ $\mathrm{ml}$ Liberase TL (Roche) and handpicked in HBSS containing 0.2\% BSA and $2.8 \mathrm{mM}$ glucose. Isolated islets were immediately fixed at $4^{\circ} \mathrm{C}$ for 3 hours with $2 \%$ paraformaldehyde, $2.5 \%$ glutaraldehyde, and 3 $\mu \mathrm{M} \mathrm{CaCl}_{2}$ in $0.1 \mathrm{M}$ sodium cacodylate buffer $\mathrm{pH}$ 7.4. Samples were processed as described (62). For quantification analyses, the area occupied by insulin vesicles and the $\beta$ cell cytoplasmic area were measured with Image J using 50-60 images per mouse.

Islet diameter. Freshly isolated islets were maintained in ice-cold RPMI with 2\% normal calf serum, and images were gathered with an Olympus IX70 microscope. Individual islet area was determined using Image J, and results converted to diameter.

Statistics. Results are shown as mean \pm SEM. Differences between 2 groups were calculated using a 2-sided Student's $t$ test, ratio paired Student's $t$ test, or Mann-Whitney $U$ test as indicated. Differences between multiple groups and a control were calculated by 1-way ANOVA followed by Dunnett's multiple comparisons post hoc test. A $P$ value of less than 0.05 was considered statistically significant.

Study approval. The Columbia University Institutional Animal Care and Utilization Committee approved all animal procedures. Human islets were harvested from deceased donors without any identifying information. Informed consent and IRB approval were obtained at islet isolation centers.

\section{Author contributions}

$\mathrm{AB}$ conducted experiments and contributed to the experimental design, discussion, and writing of the manuscript. CZ conducted experiments and contributed to the review and editing of the manuscript. LS contributed to the experimental design, discussion, and review and editing of the manuscript. UBP contributed to the experimental design, discussion, and writing of the manuscript.

\section{Acknowledgments}

We thank A. Flete and T. Kolar for excellent technical support, as well as members of the Pajvani and Accili laboratories for insightful discussion. This work was supported by NIH DK103818 (to UBP), a Russell Berrie Foundation Award (AB), and American Diabetes Association grant 1-17-PMF-025 (AB). These studies used the resources of the Diabetes and Endocrinology Research Center Flow Core Facility funded in part through center grant 5P30DK063608.

Address correspondence to: Utpal B. Pajvani, Columbia University Medical Center, Russ Berrie Science Pavilion, 1150 St. Nicholas Avenue, New York, New York 10032, USA. Phone: 212.851.4886; Email: up2104@columbia.edu.
1. Alejandro EU, Gregg B, Blandino-Rosano M, Cras-Méneur C, Bernal-Mizrachi E. Natural history of $\beta$-cell adaptation and failure in type 2 diabetes. Mol Aspects Med. 2015;42:19-41.

2. Butler AE, Janson J, Bonner-Weir S, Ritzel R, Rizza RA, Butler PC. Beta-cell deficit and increased beta-cell apoptosis in humans with type 2 diabetes. Diabetes. 2003;52(1):102-110.

3. Talchai C, Xuan S, Lin HV, Sussel L, Accili D. Pancreatic $\beta$ cell dedifferentiation as a mechanism of diabetic $\beta$ cell failure. Cell. 2012;150(6):1223-1234 .

4. Apelqvist A, et al. Notch signalling controls pancreatic cell differentiation. Nature. 1999;400(6747):877-881.

5 . Jensen J, et al. Control of endodermal endocrine development by Hes-1. Nat Genet. 2000;24(1):36-44.

6. Fujikura J, et al. Notch/Rbp-j signaling prevents premature endocrine and ductal cell differentiation in the pancreas. Cell Metab. 2006;3(1):59-65.

7. Murtaugh LC, Stanger BZ, Kwan KM, Melton DA. Notch signaling controls multiple steps of pancreatic differentiation. Proc Natl Acad Sci US A. 2003;100(25):14920-14925.

8. Zeggini E, et al. Meta-analysis of genome-wide association data and large-scale replication identifies additional susceptibility loci for type 2 diabetes. Nat Genet. 2008;40(5):638-645.

9. Valenti L, et al. Hepatic notch signaling correlates with insulin resistance and nonalcoholic fatty liver disease. Diabetes. 2013;62(12):4052-4062.

10. Pajvani UB, et al. Inhibition of Notch signaling ameliorates insulin resistance in a FoxO1-dependent manner. Nat Med. 2011;17(8):961-967.

11. Pajvani UB, Qiang L, Kangsamaksin T, Kitajewski J, Ginsberg HN, Accili D. Inhibition of Notch uncouples Akt activation from hepatic lipid accumulation by decreasing mTorc1 stability. Nat Med. 2013;19(8):1054-1060.

12. Duncan AW, et al. Integration of Notch and Wnt signaling in hematopoietic stem cell maintenance. Nat Immunol. 2005;6(3):314-322.

13. Nowotschin S, Xenopoulos P, Schrode N, Hadjantonakis AK. A bright single-cell resolution live imaging reporter of Notch signaling in the mouse. BMC Dev Biol. 2013;13:15.

14. Kopinke D, Brailsford M, Shea JE, Leavitt R, Scaife CL, Murtaugh LC. Lineage tracing reveals the dynamic contribution of Hes1+ cells to the developing and adult pancreas. Development. 2011;138(3):431-441.

15. Billiard F, et al. Delta-like ligand-4-Notch signaling inhibition regulates pancreatic islet function and insulin secretion. Cell Rep. 2018;22(4):895-904.

16. Herrera PL. Adult insulin- and glucagon-producing cells differentiate from two independent cell lineages. Development. 2000;127(11):2317-2322.

17. Tamarina NA, Roe MW, Philipson L. Characterization of mice expressing Ins1 gene promoter driven CreERT recombinase for conditional gene dele- tion in pancreatic $\beta$-cells. Islets. 2014;6(1):e27685.

18. Kopan R, Schroeter EH, Weintraub H, Nye JS. Signal transduction by activated mNotch: importance of proteolytic processing and its regulation by the extracellular domain. Proc Natl Acad Sci US A. 1996;93(4):1683-1688.

19. Han SI, Aramata S, Yasuda K, Kataoka K. MafA stability in pancreatic beta cells is regulated by glucose and is dependent on its constitutive phosphorylation at multiple sites by glycogen synthase kinase 3. Mol Cell Biol. 2007;27(19):6593-6605.

20. Rocques N, et al. GSK-3-mediated phosphorylation enhances Maf-transforming activity. $\mathrm{Mol}$ Cell. 2007;28(4):584-597.

21. Guo S, et al. The stability and transactivation potential of the mammalian MafA transcription factor are regulated by serine 65 phosphorylation. J Biol Chem. 2009;284(2):759-765.

22. Kurooka H, Honjo T. Functional interaction between the mouse notch1 intracellular region and histone acetyltransferases PCAF and GCN5 J Biol Chem. 2000;275(22):17211-17220.

23. Klochendler A, et al. The genetic program of pancreatic $\beta$-cell replication in vivo. Diabetes. 2016;65(7):2081-2093.

24. Eychène A, Rocques N, Pouponnot C. A new MAFia in cancer. Nat Rev Cancer. 2008;8(9):683-693.

25. Raum JC, et al. FoxA2, Nkx2.2, and PDX-1 
regulate islet beta-cell-specific mafA expression through conserved sequences located between base pairs -8118 and -7750 upstream from the transcription start site. Mol Cell Biol. 2006;26(15):5735-5743.

26. Oropeza D, et al. Phenotypic characterization of MIP-CreERT1Lphi mice with transgene-driven islet expression of human growth hormone. Diabetes. 2015;64(11):3798-3807.

27. Tschen SI, Dhawan S, Gurlo T, Bhushan A. Age-dependent decline in beta-cell proliferation restricts the capacity of beta-cell regeneration in mice. Diabetes. 2009;58(6):1312-1320.

28. Puri S, Akiyama H, Hebrok M. VHL-mediated disruption of Sox 9 activity compromises $\beta$-cell identity and results in diabetes mellitus. Genes Dev. 2013;27(23):2563-2575.

29. Sasson A, et al. Islet pericytes are required for $\beta$-cell maturity. Diabetes. 2016;65(10):3008-3014.

30. Cras-Méneur C, Conlon M, Zhang Y, Pasca Di Magliano M, Bernal-Mizrachi E. Early pancreatic islet fate and maturation is controlled through RBP-Jк. Sci Rep. 2016;6:26874.

31. Landsman L, Parent A, Hebrok M. Elevated Hedgehog/Gli signaling causes beta-cell dedifferentiation in mice. Proc Natl Acad Sci U S A. 2011;108(41):17010-17015.

32. Szabat M, et al. Reduced insulin production relieves endoplasmic reticulum stress and induces $\beta$ cell proliferation. Cell Metab. 2016;23(1):179-193.

33. Bar Y, Russ HA, Knoller S, Ouziel-Yahalom L, Efrat S. HES-1 is involved in adaptation of adult human beta-cells to proliferation in vitro. Diabetes. 2008;57(9):2413-2420.

34. Bar Y, Russ HA, Sintov E, Anker-Kitai L, Knoller S, Efrat S. Redifferentiation of expanded human pancreatic $\beta$-cell-derived cells by inhibition of the NOTCH pathway.J Biol Chem. 2012;287(21):17269-17280.

35. van der Meulen T, et al. Virgin beta cells persist throughout life at a neogenic niche within pancreatic islets. Cell Metab. 2017;25(4):911-926.e6.

36. Hang Y, et al. The MafA transcription factor becomes essential to islet $\beta$-cells soon after birth. Diabetes. 2014;63(6):1994-2005.

37. Kitamura YI, et al. FoxO1 protects against pancreatic beta cell failure through NeuroD and MafA induction. Cell Metab. 2005;2(3):153-163.

38. Zhang $\mathrm{C}$, et al. MafA is a key regulator of glucose-stimulated insulin secretion. Mol Cell Biol. 2005;25(12):4969-4976.

39. Nishimura W, Bonner-Weir S, Sharma A. Expression of MafA in pancreatic progenitors is detrimental for pancreatic development. Dev Biol. 2009;333(1):108-120.

40. Aguayo-Mazzucato C, et al. Mafa expression enhances glucose-responsive insulin secretion in neonatal rat beta cells. Diabetologia. 2011;54(3):583-593.

41. Hamze Z, et al. Altered MENIN expression disrupts the MAFA differentiation pathway in insulinoma. Endocr Relat Cancer. 2013;20(6):833-848.

42. Pechhold S, et al. Transcriptional analysis of intracytoplasmically stained, FACS-purified cells by high-throughput, quantitative nuclease protection. Nat Biotechnol. 2009;27(11):1038-1042.

43. Banerjee RR, et al. Gestational diabetes mellitus from inactivation of prolactin receptor and $\mathrm{MafB}$ in islet $\beta$-cells. Diabetes. 2016;65(8):2331-2341.

44. Nishimura W, et al. A switch from MafB to MafA expression accompanies differentiation to pancreatic beta-cells. Dev Biol. 2006;293(2):526-539.

45. Artner I, et al. MafA and MafB regulate genes critical to beta-cells in a unique temporal manner. Diabetes. 2010;59(10):2530-2539.

46. Hagman DK, Hays LB, Parazzoli SD, Poitout V. Palmitate inhibits insulin gene expression by altering PDX-1 nuclear localization and reducing MafA expression in isolated rat islets of Langerhans. J Biol Chem. 2005;280(37):32413-32418.

47. Harmon JS, Stein R, Robertson RP. Oxidative stress-mediated, post-translational loss of MafA protein as a contributing mechanism to loss of insulin gene expression in glucotoxic beta cells. JBiol Chem. 2005;280(12):11107-11113.

48. Matsuoka TA, et al. Regulation of MafA expression in pancreatic beta-cells in $\mathrm{db} / \mathrm{db}$ mice with diabetes. Diabetes. 2010;59(7):1709-1720.

49. Bader $\mathrm{E}$, et al. Identification of proliferative and mature $\beta$-cells in the islets of Langerhans. Nature. 2016;535(7612):430-434.

50. Rui J, Deng S, Arazi A, Perdigoto AL, Liu Z, Herold KC. $\beta$ Cells that resist immunological attack develop during progression of autoimmune diabetes in
NOD mice. Cell Metab. 2017;25(3):727-738.

51. Avrahami D, et al. Aging-dependent demethylation of regulatory elements correlates with chromatin state and improved $\beta$ cell function. Cell Metab. 2015;22(4):619-632.

52. Bi P, et al. Inhibition of Notch signaling promotes browning of white adipose tissue and ameliorates obesity. Nat Med. 2014;20(8):911-918.

53. Li J, Tang Y, Cai D. IKK $\beta / N F-\kappa B$ disrupts adult hypothalamic neural stem cells to mediate a neurodegenerative mechanism of dietary obesity and pre-diabetes. Nat Cell Biol. 2012;14(10):999-1012.

54. Fukuda D, et al. Notch ligand delta-like 4 blockade attenuates atherosclerosis and metabolic disorders. Proc Natl Acad Sci U S A. 2012;109(27):E1868-E1877.

55. Jeon JH, Suh HN, Kim MO, Ryu JM, Han HJ. Glucosamine-induced OGT activation mediates glucose production through cleaved Notch1 and FoxO1, which coordinately contributed to the regulation of maintenance of self-renewal in mouse embryonic stem cells. Stem Cells Dev. 2014;23(17):2067-2079.

56. Song NJ, et al. Notch1 deficiency decreases hepatic lipid accumulation by induction of fatty acid oxidation. Sci Rep. 2016;6:19377.

57. Kim K, et al. $\gamma$-Secretase inhibition lowers plasma triglyceride-rich lipoproteins by stabilizing the LDL receptor. Cell Metab. 2018;27(4):816-827.e4.

58. Groth C, Fortini ME. Therapeutic approaches to modulating Notch signaling: current challenges and future prospects. Semin Cell Dev Biol. 2012;23(4):465-472.

59. Maillard I, et al. Mastermind critically regulates Notch-mediated lymphoid cell fate decisions. Blood. 2004;104(6):1696-1702.

60. Bartolomé A, et al. Pancreatic $\beta$-cell failure mediated by $\mathrm{mTORC} 1$ hyperactivity and autophagic impairment. Diabetes. 2014;63(9):2996-3008.

61. Bartolome A, Guillen C, Benito M. Autophagy plays a protective role in endoplasmic reticulum stress-mediated pancreatic $\beta$ cell death. Autophagy. 2012;8(12):1757-1768.

62. Koyanagi M, et al. Ablation of TSC2 enhances insulin secretion by increasing the number of mitochondria through activation of mTORC1. PLoS One. 2011;6(8):e23238. 